\title{
Stochastic Bifurcations and Noise-Induced Chaos in 3D Neuron Model
}

DOI:

$10.1142 / \mathrm{S} 0218127416300329$

\section{Document Version}

Accepted author manuscript

Link to publication record in Manchester Research Explorer

\section{Citation for published version (APA):}

Bashkirtseva, I., Fedotov, S., Ryashko, L., \& Slepukhina, E. (2016). Stochastic Bifurcations and Noise-Induced Chaos in 3D Neuron Model. International Journal of Bifurcation and Chaos in Applied Sciences and Engineering, 26(12). https://doi.org/10.1142/S0218127416300329

\section{Published in:}

International Journal of Bifurcation and Chaos in Applied Sciences and Engineering

\section{Citing this paper}

Please note that where the full-text provided on Manchester Research Explorer is the Author Accepted Manuscript or Proof version this may differ from the final Published version. If citing, it is advised that you check and use the publisher's definitive version.

\section{General rights}

Copyright and moral rights for the publications made accessible in the Research Explorer are retained by the authors and/or other copyright owners and it is a condition of accessing publications that users recognise and abide by the legal requirements associated with these rights.

\section{Takedown policy}

If you believe that this document breaches copyright please refer to the University of Manchester's Takedown Procedures [http://man.ac.uk/04Y6Bo] or contact uml.scholarlycommunications@manchester.ac.uk providing relevant details, so we can investigate your claim.

\section{OPEN ACCESS}




\title{
STOCHASTIC BIFURCATIONS AND NOISE-INDUCED CHAOS IN 3D NEURON MODEL
}

\author{
IRINA BASHKIRTSEVA \\ Institute of Mathematics and Computer Science, Ural Federal University, Lenina, 51, \\ Ekaterinburg, 620083, Russia \\ Irina.Bashkirtseva@urfu.ru \\ SERGEI FEDOTOV \\ School of Mathematics, The University of Manchester, Oxford Road, Manchester M13 9PL, UK \\ Sergei.Fedotov@manchester.ac.uk \\ LEV RYASHKO \\ Institute of Mathematics and Computer Science, Ural Federal University, Lenina, 51, \\ Ekaterinburg, 620083, Russia \\ Lev.Ryashko@urfu.ru \\ EVDOKIA SLEPUKHINA \\ Institute of Mathematics and Computer Science, Ural Federal University, Lenina, 51, \\ Ekaterinburg, 620083, Russia \\ Evdokia.Slepukhina@urfu.ru \\ Received (to be inserted by publisher)
}

\begin{abstract}
The stochastically forced three-dimensional Hindmarsh-Rose model of neural activity is considered. We study the effect of random disturbances in parametric zones where the deterministic model exhibits mono- and bistable dynamic regimes with period-adding bifurcations of oscillatory modes. It is shown that in both cases the phenomenon of noise-induced bursting is observed. In the monostable zone, where the only attractor of the system is a stable equilibrium, this effect is connected with a stochastic generation of large-amplitude oscillations due to the high excitability of the model. In a parametric zone of coexisting stable equilibria and limit cycles, bursts appear due to noise-induced transitions between the attractors. For a quantitative analysis of the noise-induced bursting and corresponding stochastic bifurcations, an approach based on the stochastic sensitivity function (SSF) technique is applied. Our estimations of the strength of noise that generates such qualitative changes in stochastic dynamics are in a good agreement with the direct numerical simulation. A relationship of the noise-induced generation of bursts with transitions from order to chaos is discussed.
\end{abstract}

Keywords: Hindmarsh-Rose model; excitability; stochastic sensitivity; noise-induced transitions; stochastic generation of bursting oscillations; stochastic bifurcations; noise-induced chaotization

\section{Introduction}

A combination of nonlinearity and stochasticity in dynamical systems can lead to unexpected effects which often have no analogues in the deterministic case. A huge variety of phenomena induced by random 
disturbances is known, such as stochastic resonance [Gammaitoni et al., 1998; McDonnell et al., 2008; Anishchenko et al., 2007], noise-induced transitions [Horsthemke \& Lefever, 1984], noise-induced chaos [Gao et al., 1999] and order [Matsumoto \& Tsuda, 1983; Gassmann, 1997], stochastic bifurcations [Arnold, 1998].

Recently, nonlinear phenomena in models of neural activity attract attention of both mathematicians and biologists [Izhikevich, 2007]. The 3D Hindmarsh-Rose (HR) model [Hindmarsh \& Rose, 1984] describes a wide range of neural dynamic regimes, namely various periodic oscillations resulting from the perioddoubling and adding bifurcations, coexistence of several attractors, chaos. A detailed bifurcation analysis of the deterministic HR model is presented, for example, in [Innocenti et al., 2007; Shilnikov \& Kolomiets, 2008; Storace et al., 2008; Barrio \& Shilnikov, 2011].

One of the important types of the neural activity is bursting: a mode when intervals of the periodic spiking alternate with intervals of the resting [Izhikevich, 2007]. Mathematical mechanisms underlying the bursting dynamics in HR model were studied in [Wang, 1993]. Chaotic transitions between spiking and bursting solutions were investigated in [Gonzalez-Miranda, 2003; Innocenti et al., 2007]. Bifurcation scenarios giving rise to irregular or chaotic spiking and bursting were analysed in [Barrio et al., 2014]. Mixed-mode bursting oscillations were discussed in [Desroches et al., 2013].

Noise-induced phenomena in neural models attract attention of many researchers [Pikovsky \& Kurths, 1997; Lindner \& Schimansky-Geier, 1999; Bashkirtseva et al., 2014; Wang et al., 2016]. The stochastic variant of the HR model was investigated by several authors. Primarily, effects of noise on the system with periodic external impulse and stochastic resonance [Longtin, 1997; Reinker et al., 2003; Wang et al., 2000; Osipov \& Ponizovskaya, 2000; Ji \& Bi, 2011; Baltanas \& Casado, 2002] were studied. Coherence resonance in stochastic HR model was analysed in [Shi \& Lu, 2005; Gu et al., 2002].

The aim of our research is to study the influence of noise on the dynamics of the HR model. In particular, we analyse a stochastic generation of bursting oscillations and noise-induced transitions between order and chaos. For the analysis of such phenomena, we suggest an approach based on the stochastic sensitivity function technique and the method of confidence domains.

The present paper is organized as follows.

In Sec. 2, a brief overview of attractors and bifurcations of the deterministic HR model is given.

Our paper is focused on the study of the effects of random disturbances on the HR model. At first, in Subsec. 3.1, we consider a parametric zone, where the stable equilibrium is a single attractor of the deterministic system. We show that even in this seemingly simple case, under the noise, the HR model exhibits rather complex phenomenon of the stochastic generation of bursting. For a weak noise, random states concentrate near the equilibrium. With an increase of the noise intensity, random trajectories can go far from the stable equilibrium, and along with small-amplitude oscillations around the equilibrium, bursts are observed. An underlying reason of such stochastic bifurcation is in a high excitability of the HR model.

In Subsec. 3.2, a parametric region, where the equilibrium coexists with the limit cycle, is studied. Here, random disturbances can induce transitions from one basin of attraction to another, and generate the bursting type behavior.

A basic mathematical model for theoretical analysis of stochastic dynamics in terms of probability density function is Kolmogorov-Fokker-Planck (KFP) equation. It gives the most detailed probabilistic description of the stochastic attractors and stochastic bifurcations. However, a direct usage of this equation is very difficult technically even in simple cases. For an approximation of KFP solutions, a well-known quasipotential method [Freidlin \& Wentzell, 1984; Dembo \& Zeitouni, 1995] and a stochastic sensitivity function technique [Bashkirtseva \& Ryashko, 2005, 2004] can be used.

Stochastic sensitivity function (SSF) technique [Bashkirtseva \& Ryashko, 2011] allows us to construct confidence domains that are simple and evident geometrical models for a spatial description of a configurational arrangement of random states around the deterministic attractors. In Subsec. 3.1 and 3.2, for mono- and bistability zones, noise-induced transitions and stochastic generation of bursting oscillations in HR model are analysed using the stochastic sensitivity function technique.

The phenomena of stochastic generation of large-amplitude oscillations and noise-induced transitions are accompanied with changes in number of spikes in a burst. In Subsec. 3.3, results of the probabilistic 
analysis of these phenomena are given.

Noise-induced transitions from order to chaos in connection with the stochastic generation of bursting oscillations are discussed in Sec. 4.

\section{Deterministic model}

The three-dimensional Hindmarsh-Rose (HR) [Hindmarsh \& Rose, 1984] model of the neural activity is given by a following system of differential equations:

$$
\begin{aligned}
& \dot{x}=y-x^{3}+3 x^{2}+I-z \\
& \dot{y}=1-5 x^{2}-y \\
& \dot{z}=r\left(s\left(x-x_{0}\right)-z\right),
\end{aligned}
$$

where $x$ is a membrane potential, variables $y, z$ describe ionic currents, $I$ is an external current; $0<r \ll 1$ is a time scale parameter; $s, x_{0}$ are other parameters.

Here we fix $r=0.002, s=4, x_{0}=-1.6$.

Consider the dynamics of the system (1) under variation of the parameter $I$. Fig. 1 shows a bifurcation diagram of the deterministic system. Here, $z$-coordinates of equilibrium points and $z$-coordinates of points of intersection of limit cycles and chaotic attractors with Poincare section plane $x=0$ in dependence of the parameter $I$ value are plotted.

The system has a unique equilibrium point. For low current values $\left(I<I_{1} \approx 1.288\right)$, it is stable. The equilibrium loses its stability due to the subcritical Hopf bifurcation at the point $I_{1}$.

For $I<I_{0} \approx 1.2677$, the stable equilibrium is a unique attractor of the system. At the point $I_{0}$, a limit cycle emerges via saddle-node bifurcation. A type of this limit cycle is termed by "burst". The bursting activity is observed in the system for $I_{0}<I<I_{2} \approx 3.292$. For $I_{0}<I<I_{1}$, the system exhibits a coexistence of a stable limit cycle and a stable equilibrium. Initially, near $I_{0}$, a limit cycle has only one spike in a burst. Then, with the increase of $I$, via period-adding bifurcations, bursts with $2,3,4, \ldots, 13$ spikes appear. A transition from burst with 12 spikes to burst with 13 spikes is accompanied with the transition to chaos [Innocenti et al., 2007; Gonzalez-Miranda, 2003; Shilnikov \& Kolomiets, 2008].

Fig. 2 shows examples of the burst with one spike for $I=1.268$ (Fig. 2a), and the burst with two spikes for $I=1.28$ (Fig. 2b).

For $I_{2}<I<I_{6} \approx 25.261$, the system exhibits tonic spiking solutions. For $I_{2}<I<I_{3} \approx 3.37$, a period-doubling cascade of bifurcations with a transition to chaos occurs.

The equilibrium becomes stable again at $I_{4} \approx 5.398$ via the supercritical Hopf bifurcation, and for $I_{4}<I<I_{5} \approx 6.198$ there is a coexistence of a stable equilibrium and a stable spiking cycle. At $I_{4}$ the equilibrium loses its stability again due to the subcritical Hopf bifurcation.

The limit cycle disappears at $I_{6}$ where the supercritical Hopf bifurcation takes place. For $I>I_{6}$, the stable equilibrium is a unique attractor of the system.

\section{Stochastic model}

Consider the stochastic HR model:

$$
\begin{aligned}
& \dot{x}=y-x^{3}+3 x^{2}+I-z+\varepsilon \dot{w}, \\
& \dot{y}=1-5 x^{2}-y \\
& \dot{z}=r\left(s\left(x-x_{0}\right)-z\right),
\end{aligned}
$$

where $w$ is a standard Wiener process with $\mathrm{E}(w(t)-w(s))=0, \mathrm{E}(w(t)-w(s))^{2}=|t-s|$ and $\varepsilon$ is a noise intensity.

In this paper, we focus on stochastic phenomena in the parametric zone $I \in(1.2,1.288)$, where the unforced deterministic system (1) exhibits two dynamical regimes: monostable but excitable regime with the only stable equilibrium (for $I<I_{0}$ ), and bistable regime with the coexistence of the stable equilibrium and the stable limit cycle (for $I_{0}<I<I_{1}$ ). 

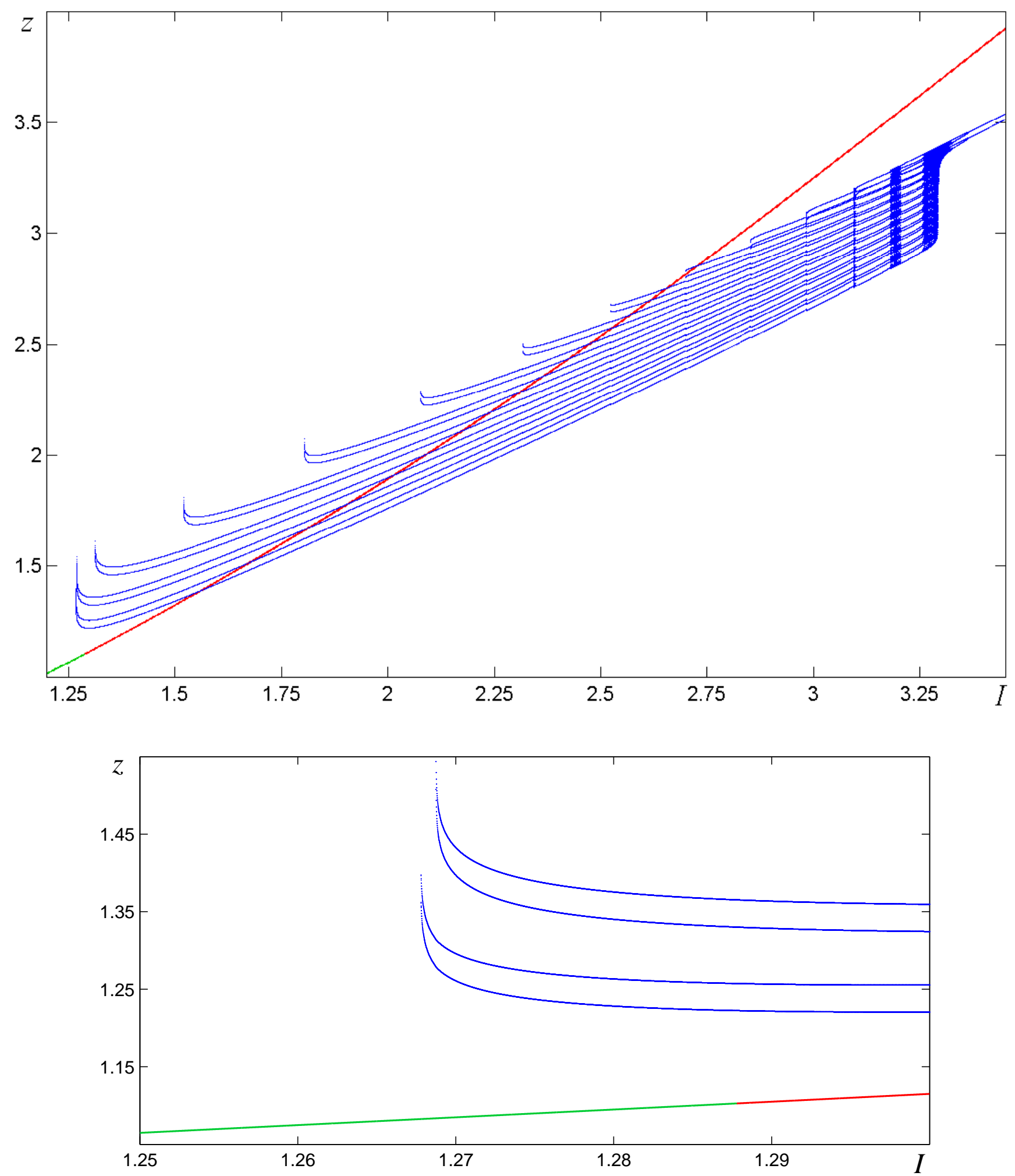

Fig. 1. Bifurcation diagram of the deterministic HR model: $z$-coordinates of equilibrium points (green for stable, and red for unstable), $z$-coordinates of points of intersection of limit cycles and chaotic attractors with Poincare section plane $x=0$ (blue). 
a)
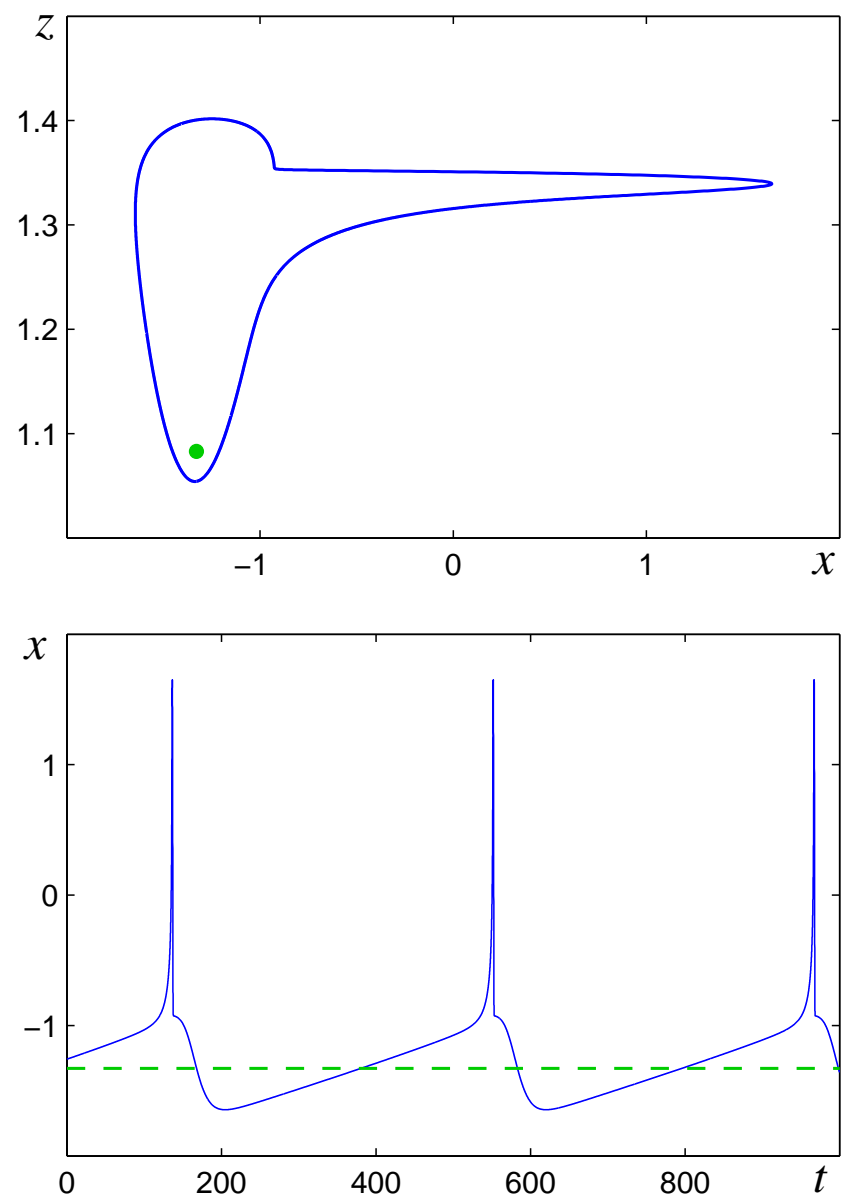

b)
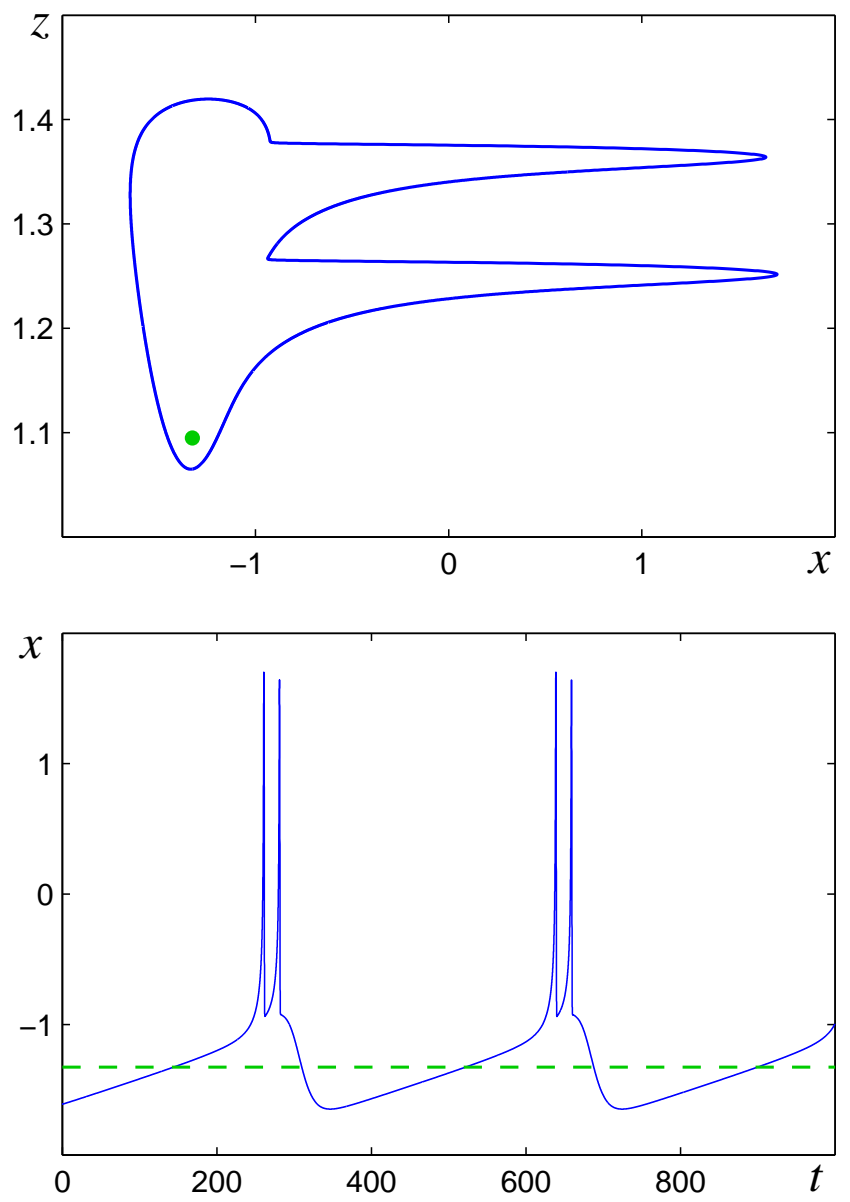

Fig. 2. Phase portraits (in projection on $x O z$ plane) and time series of the deterministic HR model for a) $I=1.268$, b) $I=1.28$ : coexisting equilibrium (green dashed) and limit cycle (blue solid).

\subsection{Excitability. Stochastic generation of bursting oscillations}

Let us study the effect of random disturbances on the system (2) in the parametric zone $I<I_{0}$, where a stable equilibrium is the only attractor of the deterministic system. For parameter values that are close to the bifurcation point $I_{0}$, the deterministic system is excitable. Indeed, small deviations from the equilibrium result in the monotonic convergence to the equilibrium. But the deviations larger than some threshold can result in a large excursion before returning to the resting state. The excitability is one of the essential properties of neuron models.

Such excitability with respect to deterministic deviations implies the corresponding stochastic excitability. In Fig. 3, for $I=1.2$, random trajectories (in projection on the plane $x O z$ ) starting from the stable equilibrium, and corresponding time series of the system (2) for different values of the noise intensity are plotted. For a sufficiently small noise $(\varepsilon=0.03)$, random trajectories oscillate near the equilibrium (see Fig. 3a). When the noise intensity is greater than some threshold value, random trajectories can go far from the stable equilibrium, and along with small-amplitude oscillations (SAO), large-amplitude oscillations (LAO) occur. Such intermittency of stochastic SAO and LAO forms the stochastic bursting (see Fig. $3 \mathrm{~b}$ for $\varepsilon=0.1$ ). One can see that a number of spikes in bursts is random.

Consider a process of the stochastic generation of bursting oscillations for different values of the parameter $I$ in the zone $I<I_{0}$. Fig. 4 shows the details of the distribution of random states in dependence on the noise intensity $\varepsilon$ for $I=1.2$ (Fig. 4a) and $I=1.25$ (Fig. 4b). Here, $z$-coordinates of points of intersection of random trajectories with the Poincare section line $x=\bar{x}(\bar{x}$ is $x$-coordinate of the 
a)
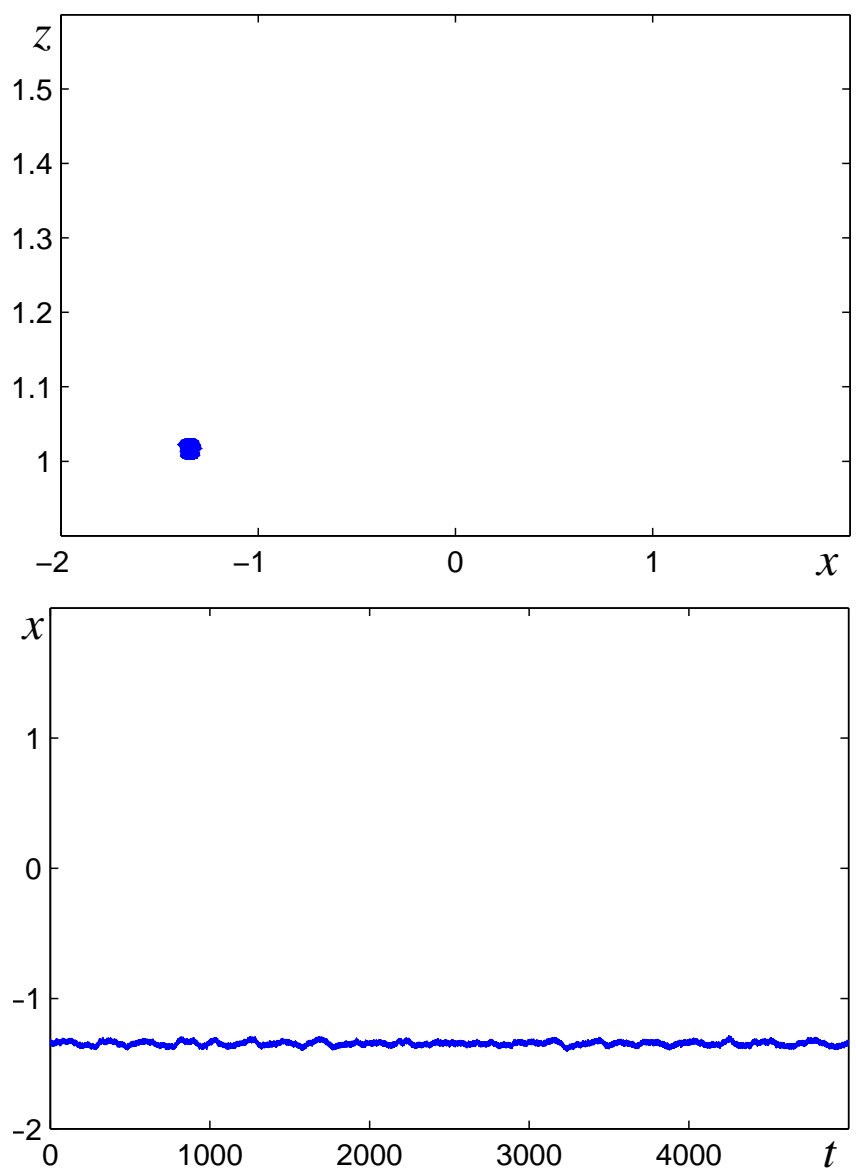

b)
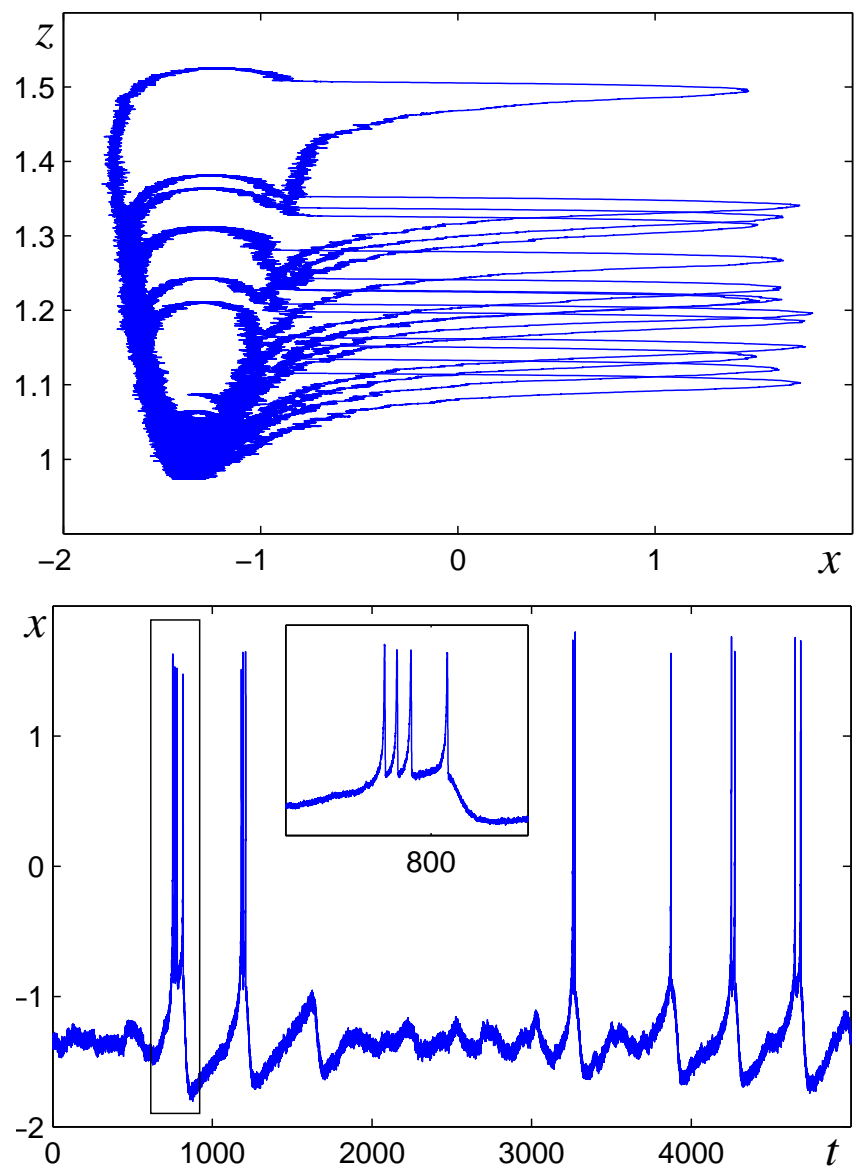

Fig. 3. Phase portraits (in projection on $x O z$ plane) and time series for $I=1.2$ : a) $\varepsilon=0.03$, b) $\varepsilon=0.1$. For a small noise (a), random trajectories oscillate near the equilibrium. For a greater noise intensity (b), and along with small-amplitude oscillations (SAO) near the equilibrium, large-amplitude oscillations (LAO) occur. Such intermittency of SAO and LAO forms the stochastic bursting regime.

equilibrium) are plotted. As one can see, for small noise intensities, random states concentrate near the equilibrium. With an increase in the noise, states with large $z$-coordinates appear, that confirms the emergence of large-amplitude trajectories. Note that for $I=1.25$ large-amplitude oscillations are observed for lower noise intensity values than for $I=1.2$, because the value $I=1.25$ is closer to the bifurcation point $I_{0}$.

Fig. 5 demonstrates changes of the probability density distribution of random trajectories. The probability density function $P(z)$ for $z$-coordinates of points of intersection of random trajectories with $x=\bar{x}$ is plotted for $I=1.2$ (Fig. 5a) and $I=1.25$ (Fig. 5b) for various noise intensities. For a weak noise, the probability density function has a single peak located above the equilibrium point. With an increase of the noise intensity, a new peak of $P(z)$ appears, so $P(z)$ function becomes bimodal. Thus, under the random disturbances, the system demonstrates $P$-bifurcation [Arnold, 1998] related to the qualitative change in the distribution of random trajectories.

Note that for large amplitude oscillations, random states with $x>-1$ are observed. The value $x=-1$ can be used as a threshold that separates in the phase space small-amplitude oscillations near equilibrium from the spiking phase. For a quantification of the weight of spiking time in the total time of the observation, consider the numerical characteristic $\eta=\frac{T_{l}}{T}$, where $T_{l}$ is the time spending by the system in the region $x>-1$ and $T$ is the total time. In Fig. 6, functions $\eta(\varepsilon)$ for different $I$ are plotted. For a small noise, random states are concentrated near the equilibrium, so $\eta=0$. For increasing noise intensity values, the large-amplitude oscillations are observed, and $\eta$ increases as well. The plot $\eta(\varepsilon)$ allows us to estimate a 
a)

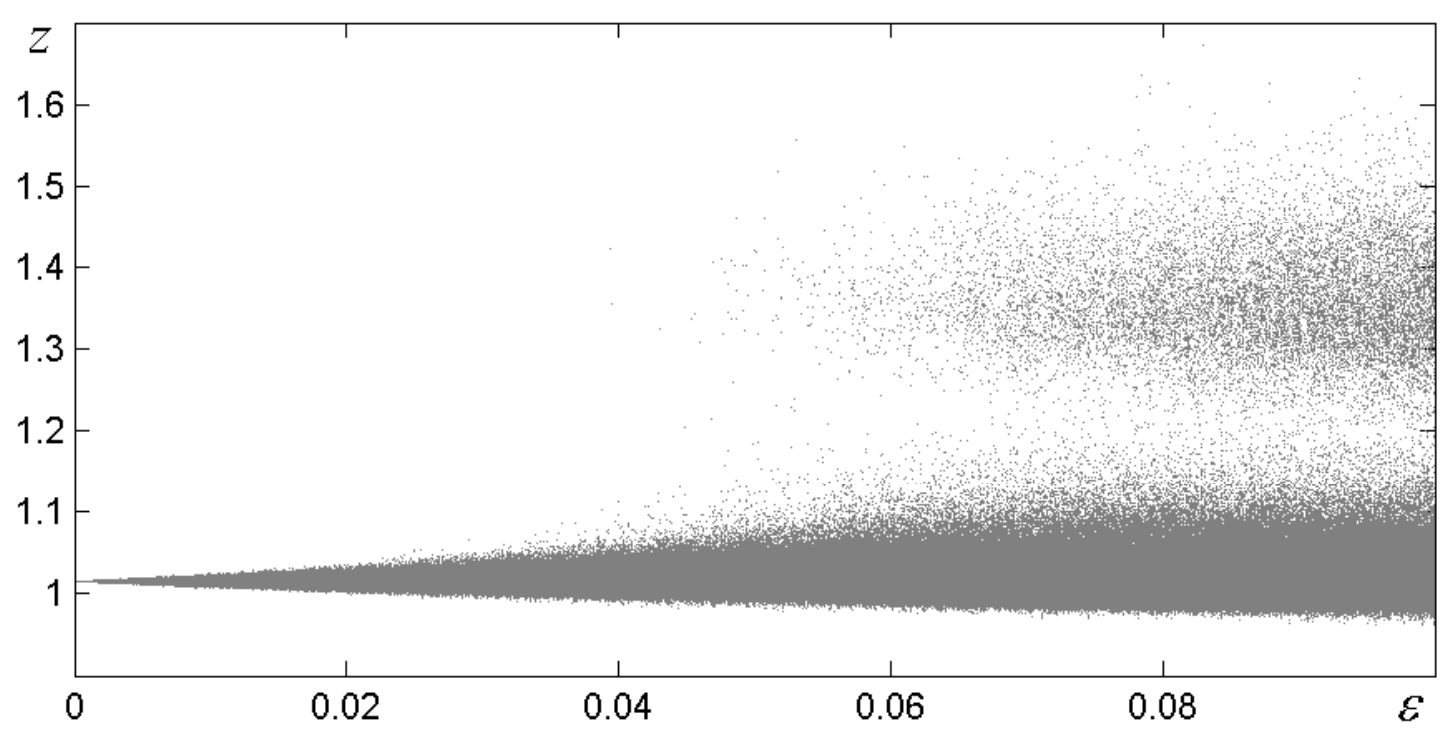

b)

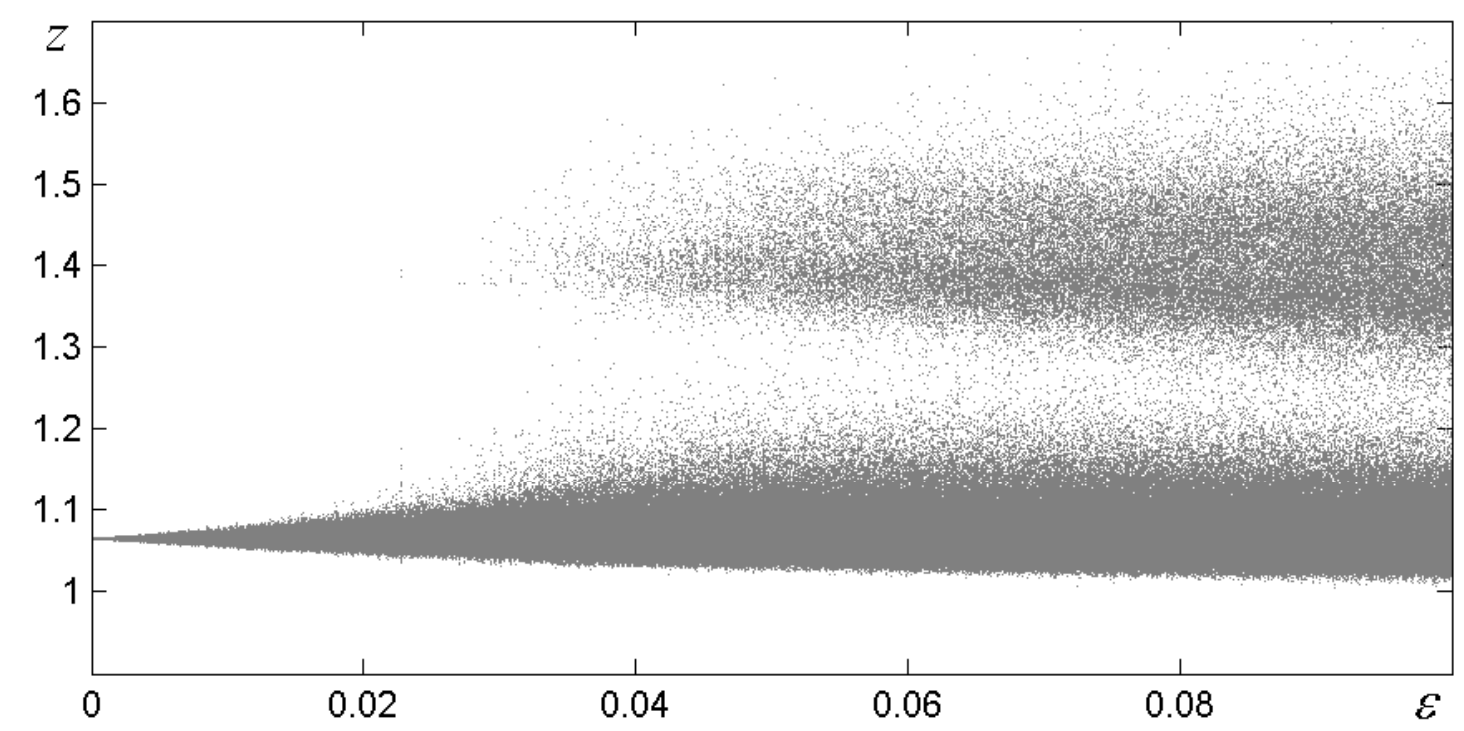

Fig. 4. Stochastic generation of large amplitude oscillations: $z$-coordinates of points of intersection of random trajectories with the Poincare section $x=\bar{x}$, a) $I=1.2$, b) $I=1.25$. For small noise intensities, random states concentrate near the equilibrium. With an increase of noise, states with large $z$-coordinates appear, corresponding to the appearance of largeamplitude oscillations and the onset of the bursting regime.

critical value of the noise intensity, corresponding to the onset of spiking generation. For $I=1.2$, we get $\varepsilon^{*} \approx 0.06$, and for $I=1.25$ we have $\varepsilon^{*} \approx 0.04$.

The emergence of noise-induced large-amplitude oscillations can be explained by peculiarities of the phase portrait of the deterministic system in zone $I<I_{0}$. In this parametric region, the equilibrium is stable. If initial states are deviated from the equilibrium, the trajectories tend to it, but a character of a transient process depends on the value of the initial deviation. Indeed, if the deviations are sufficiently small, the trajectories tend to the equilibrium monotonically. If we take the initial deviations larger than some threshold, the transient process has a spike (see Fig. 7). The further increase in the deviations result 
a)

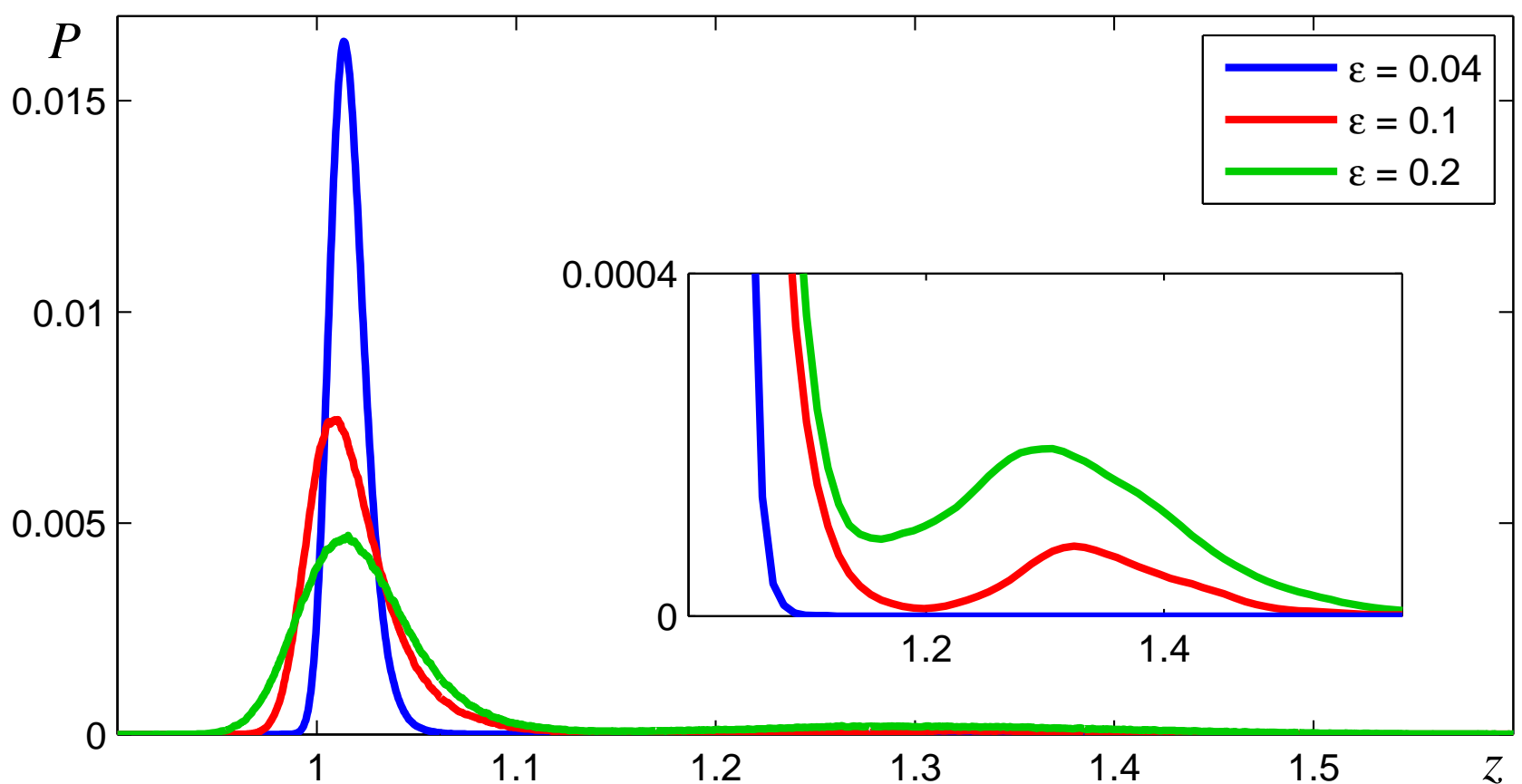

b)

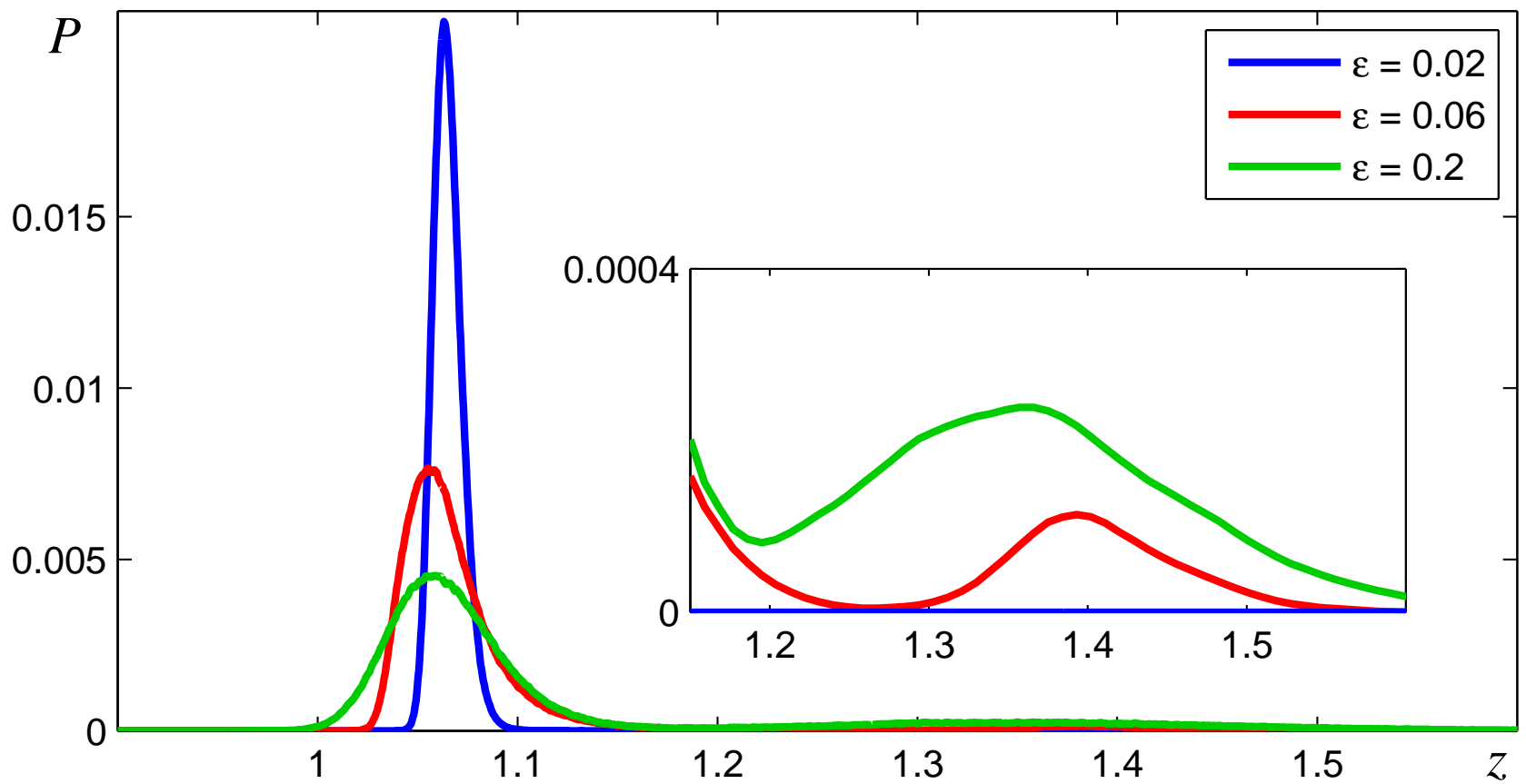

Fig. 5. Distribution of random trajectories: a) $I=1.2$, b) $I=1.25$. For a weak noise, the probability density function has a single peak located above the equilibrium point. With an increase of the noise intensity, a new peak of $P(z)$ in the zone $z>1.2$ appears. Thus, the system demonstrates $P$-bifurcation.

in the trajectories with two, three and more spikes.

To analyze the mechanism of stochastic generation of large-amplitude oscillations, we apply the stochastic sensitivity function technique. A brief theoretical background of SSF technique is given in the Appendix.

The stochastic sensitivity matrix reflects the geometry of bundles of stochastic trajectories around 


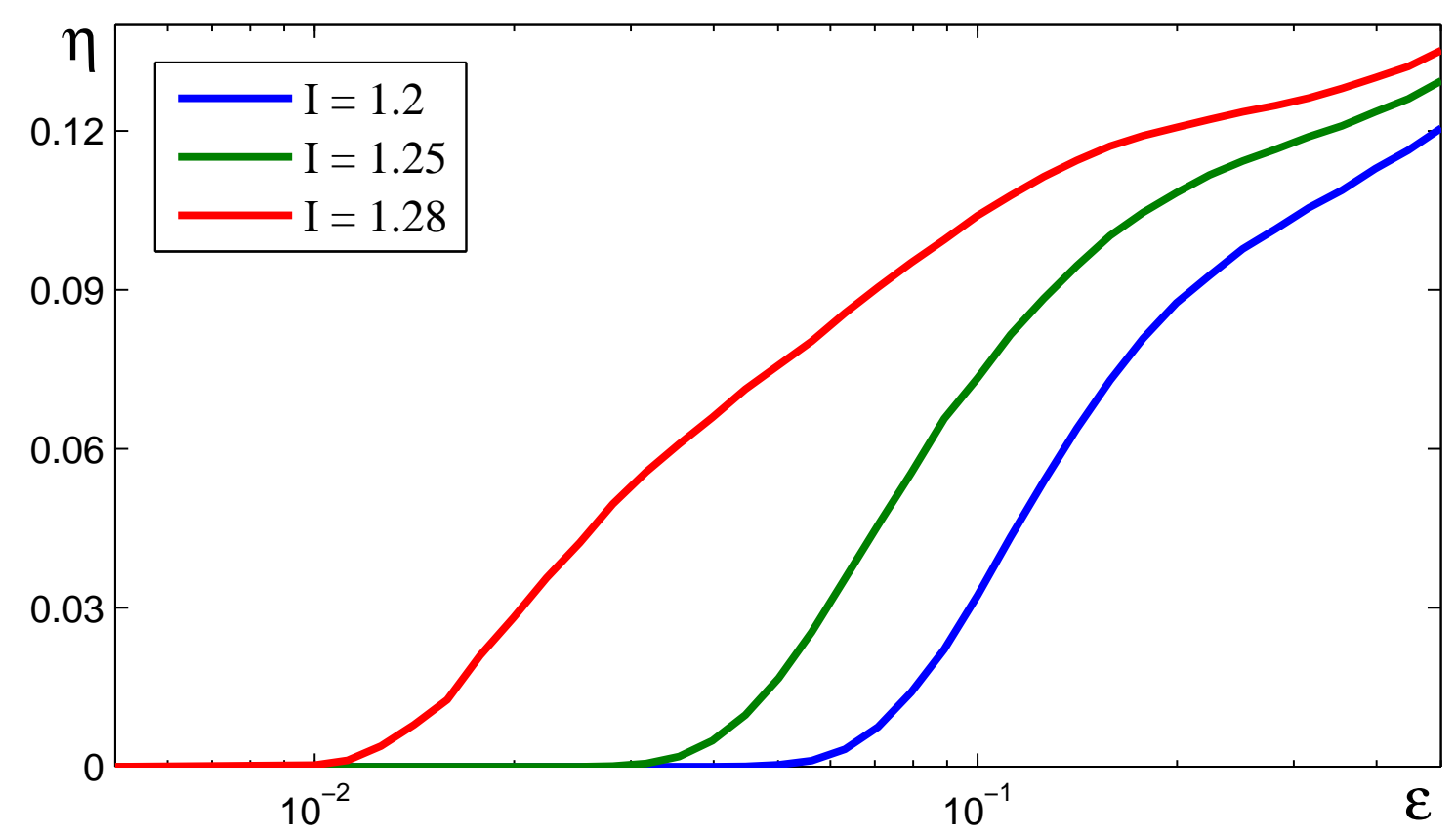

Fig. 6. Function $\eta=\frac{T_{l}}{T}$. Here, $T_{l}$ is the time spending by the system in the region $x>-1$ and $T$ is the total time. The value $x=-1$ is used as a threshold that separates in the phase space small-amplitude oscillations near equilibrium from the spiking phase. The increase of $\eta$ corresponds to the onset of spiking generation.

the deterministic attractors. If the attractor is an equilibrium, this distribution can be estimated by a confidence ellipsoid. Eigenvectors of the stochastic sensitivity matrix determine a spatial arrangement of axes of the ellipsoid while eigenvalues define a size of them.

Fig. 8 shows eigenvalues of the stochastic sensitivity matrix for the equilibrium in the parametric zone $I \in(1.2,1.288)$. One can see that the stochastic sensitivity increases unlimitedly with the approaching to the bifurcation point $I_{1}$.

Random states of the system (2) are distributed non-uniformly around the equilibrium. Note that the difference in eigenvalues of the stochastic sensitivity matrix for each fixed $I$ is significant. So the confidence ellipsoids are elongated in the direction of the eigenvector $v^{*}$ corresponding to the largest eigenvalue of the stochastic sensitivity matrix, and they are very narrow in other directions. Thus, the eigenvector $v^{*}$ localizes the main direction for deviations of random trajectories from the equilibrium.

Fig. 9 shows the projection of the line passing through the equilibrium point in the direction $v^{*}$ on the plane $x O z$, and the intervals $M M_{0}, M M_{1}, M M_{2}$, starting from the equilibrium $M$ and corresponding to zones with different number of spikes in the transient process. Trajectories starting from any point of $M M_{0}$ tend to the equilibrium monotonically without spikes. Initial points taken from $M M_{1}$ and $M M_{2}$ result in large-amplitude trajectories with one and two spikes respectively.

Using these intervals of deviations we can construct confidence intervals for the distribution of random trajectories, and estimate the critical values of the noise intensity. The noise intensity $\varepsilon_{0}$ corresponds to $M M_{0}$, and for $\varepsilon<\varepsilon_{0}$ there are small-amplitude stochastic oscillations near the equilibrium. The values $\varepsilon_{1}$ and $\varepsilon_{2}$ that can be estimated using intervals $M M_{1}$ and $M M_{2}$, correspond to the onset of the generation of bursts with one and two spike respectively.

For $I=1.2$, we get $\varepsilon_{0} \approx 0.0675, \varepsilon_{1} \approx 0.0684, \varepsilon_{2} \approx 0.1084$ and for $I=1.25$ we have $\varepsilon_{0} \approx 0.0391$, $\varepsilon_{1} \approx 0.0395, \varepsilon_{2} \approx 0.0628$. The obtained values are in a good agreement with the results of the direct numerical simulations. 
a)

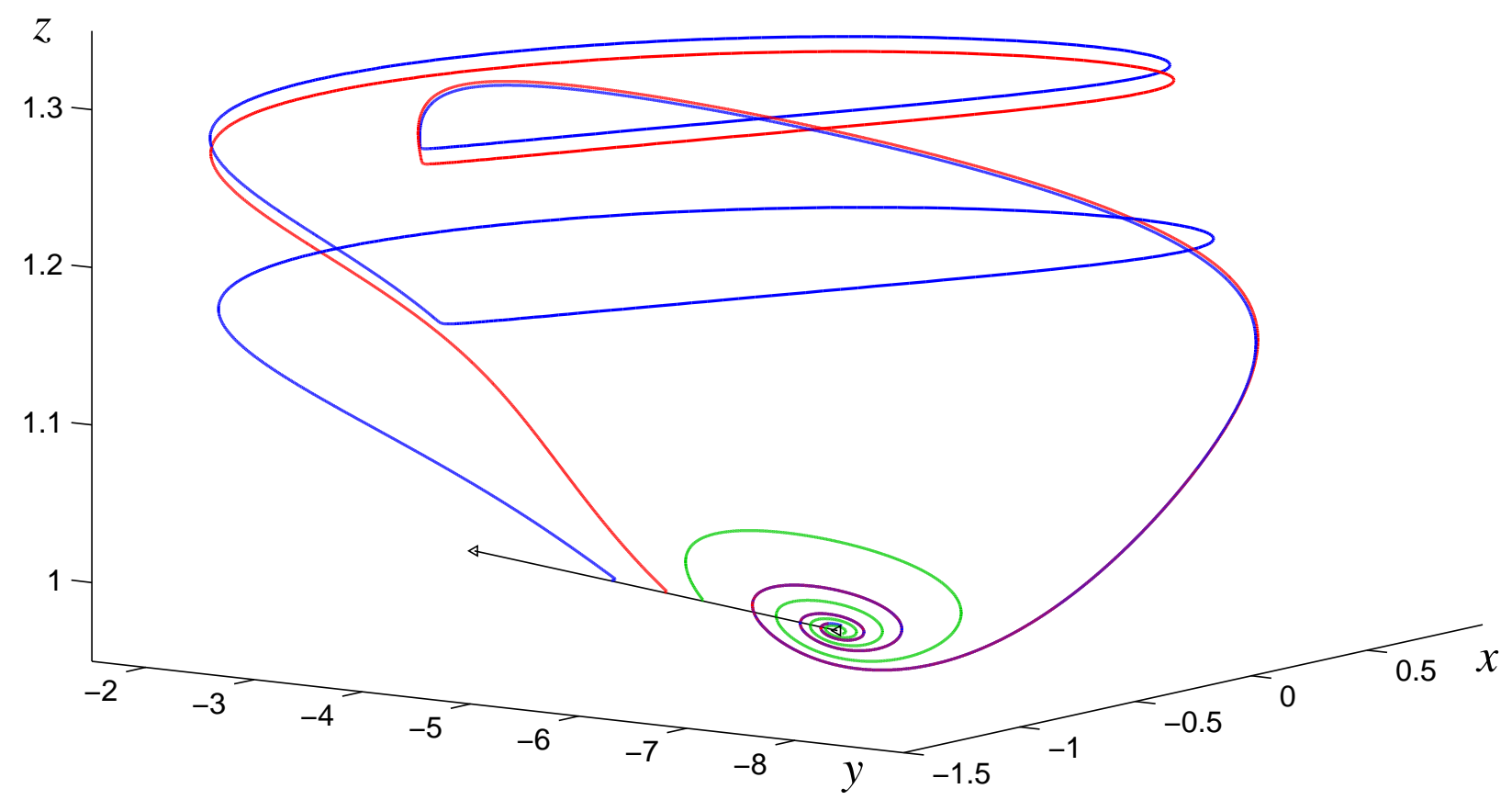

b)

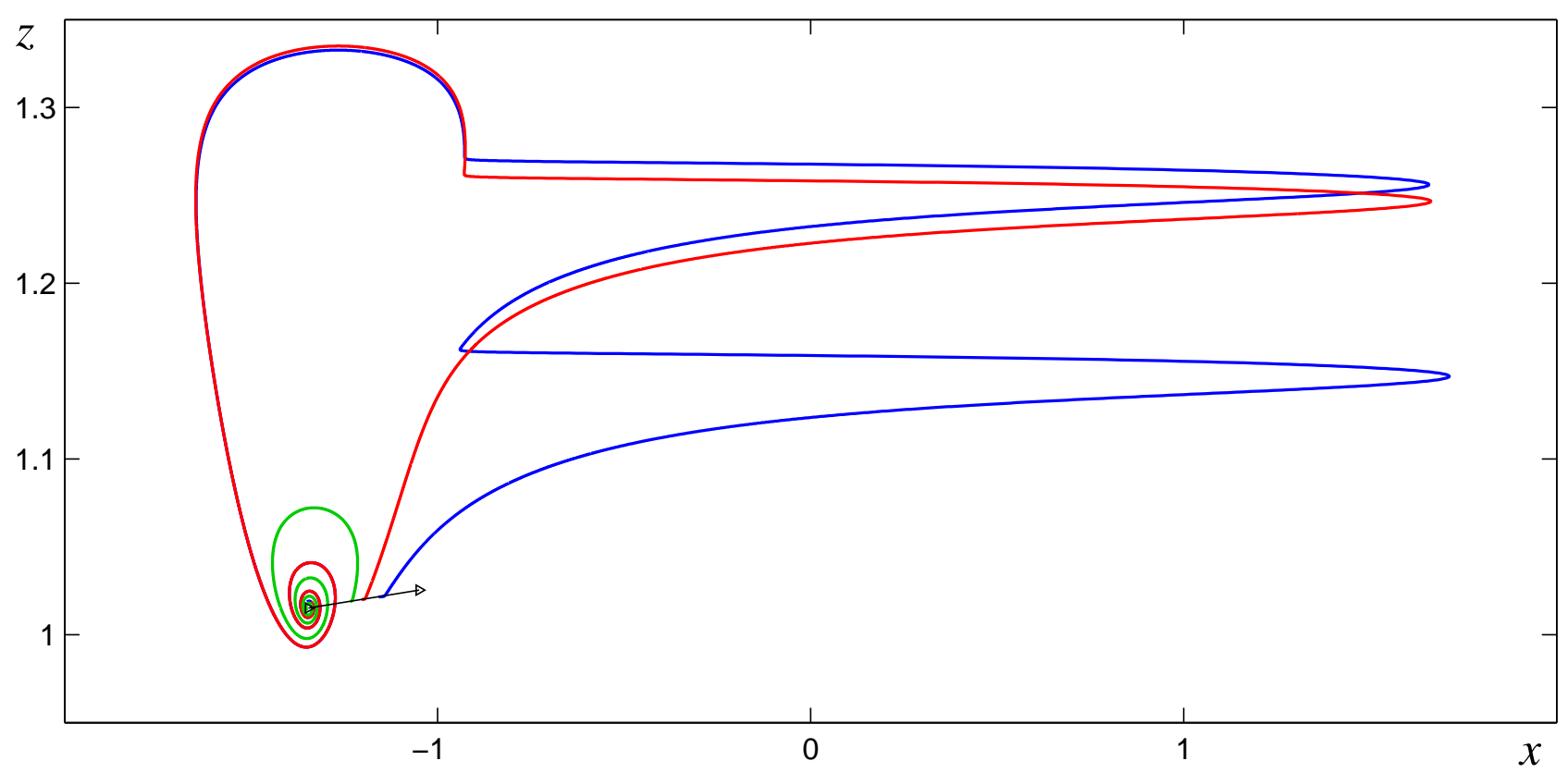

Fig. 7. Deterministic phase portraits and eigenvector corresponding to the largest eigenvalue of SSF for $I=1.2$ : a) in $x y z-$ space, b) in projection on $x O z$-plane. A character of a transient process depends on the value of the initial deviation: the trajectories tend to the equilibrium monotonically without spikes, or the transient process has one, two or more spikes.

\subsection{Bistability. Noise-induced transitions between equilibrium and limit cycle}

Let us study the effect of random disturbances on the system (2) in the parametric zone $I \in(1.268,1.288)$, where a stable equilibrium and a stable limit cycle coexist. 


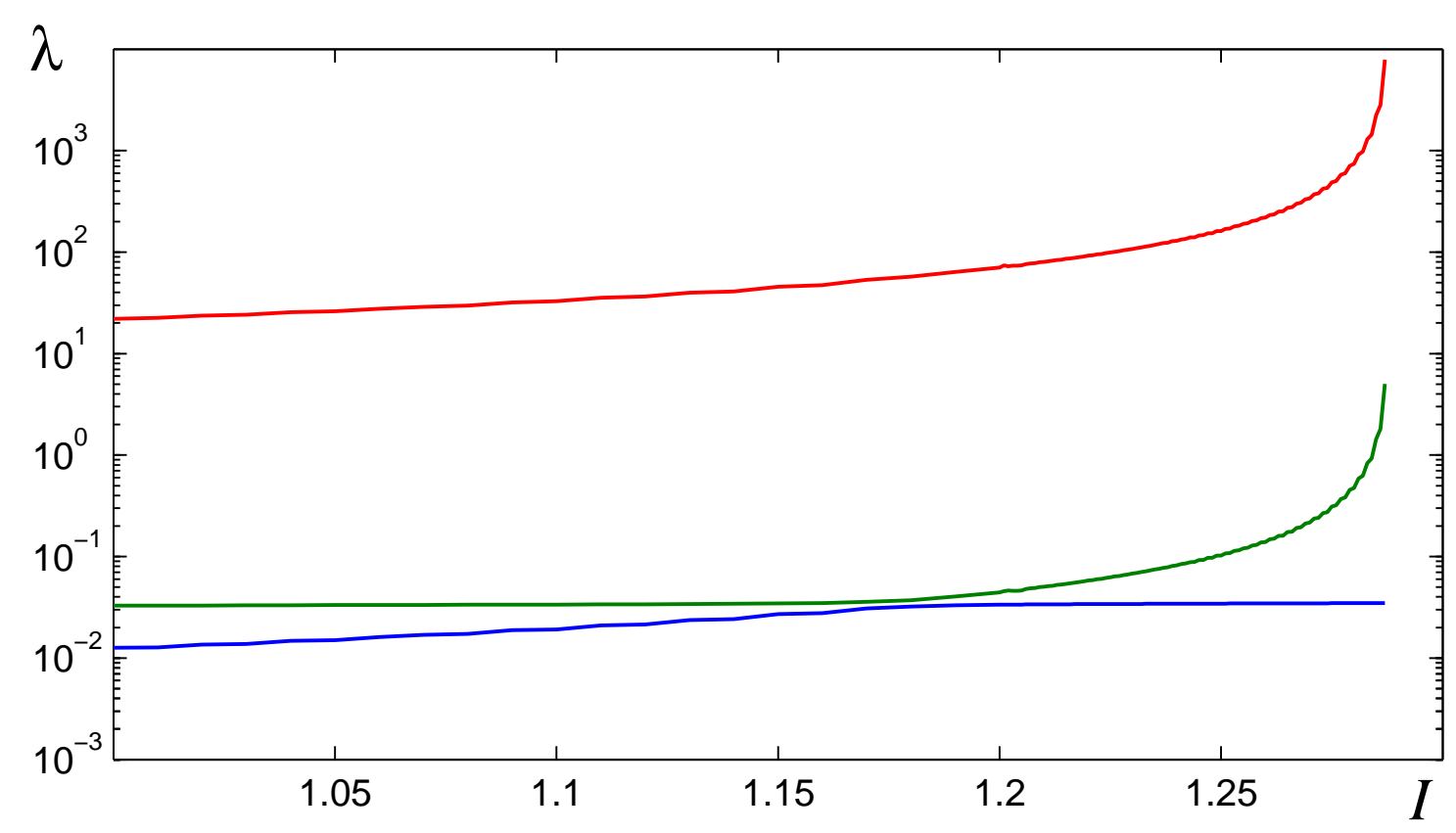

Fig. 8. Eigenvalues of the stochastic sensitivity matrix for the equilibrium. The stochastic sensitivity increases unlimitedly with the approaching to the bifurcation point $I_{1}$.

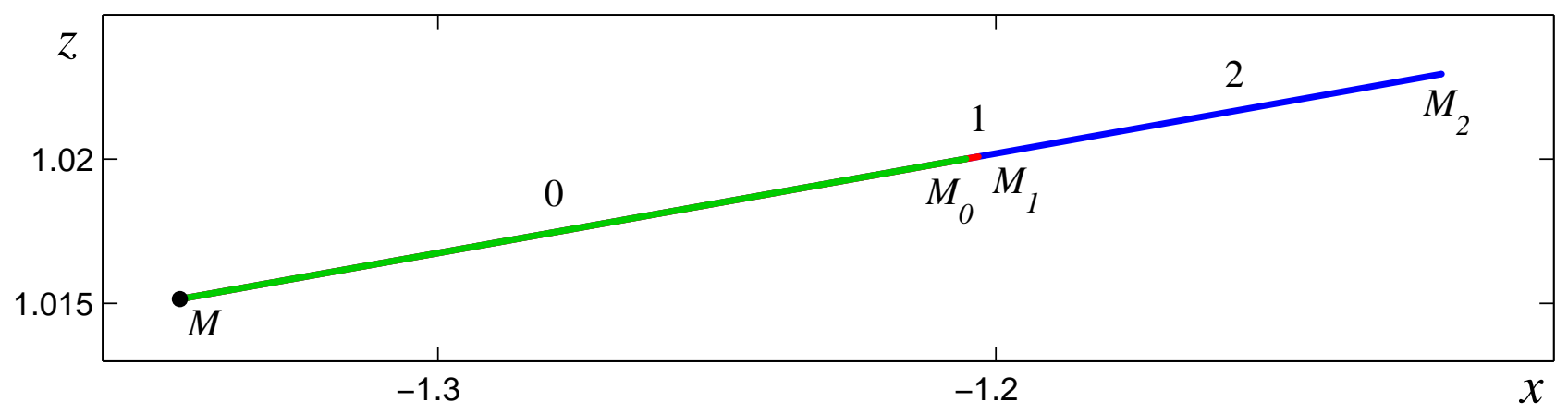

Fig. 9. The projection of the line passing through the equilibrium point in the direction of the eigenvector corresponding to the largest eigenvalue of SSF on the plane $x O z$, and the intervals corresponding to zones with different number of spikes in the transient process, for $I=1.2$.

Consider $I=1.27$. In Fig. 10, random trajectories (in projection on the plane $x O z$ ) starting from the limit cycle and corresponding time series of the system (2) for different values of the noise intensity are plotted. For sufficiently small values of noise intensity $(\varepsilon=0.0005)$, random trajectories are concentrated near the deterministic cycle (Fig. 10a). For a noise intensity value greater than some threshold, stochastic trajectories can cross the separatrix surface, separating basins of attraction of the equilibrium and the limit cycle, and go to the stable equilibrium (Fig. 10b for $\varepsilon=0.002$ ). With an increase in the noise intensity, backward transitions from the basin of attraction of the equilibrium to the basin of attraction of the limit cycle can occur (Fig. 10c for $\varepsilon=0.07$ ).

The details of the distribution of random states in dependence of noise intensity are shown in Figs. 11, 12. Consider points of intersection of the random trajectories with the Poincare section line $x=\bar{x}$. Fig. 11 shows $z$-coordinates of these points in dependence on the noise intensity for $I=1.27$ and $I=1.28$. Note that for small noise intensities, random states concentrate near the deterministic limit cycle. With an increase of noise, random states near the equilibrium appear, which corresponds to the onset of the noise- 
a)
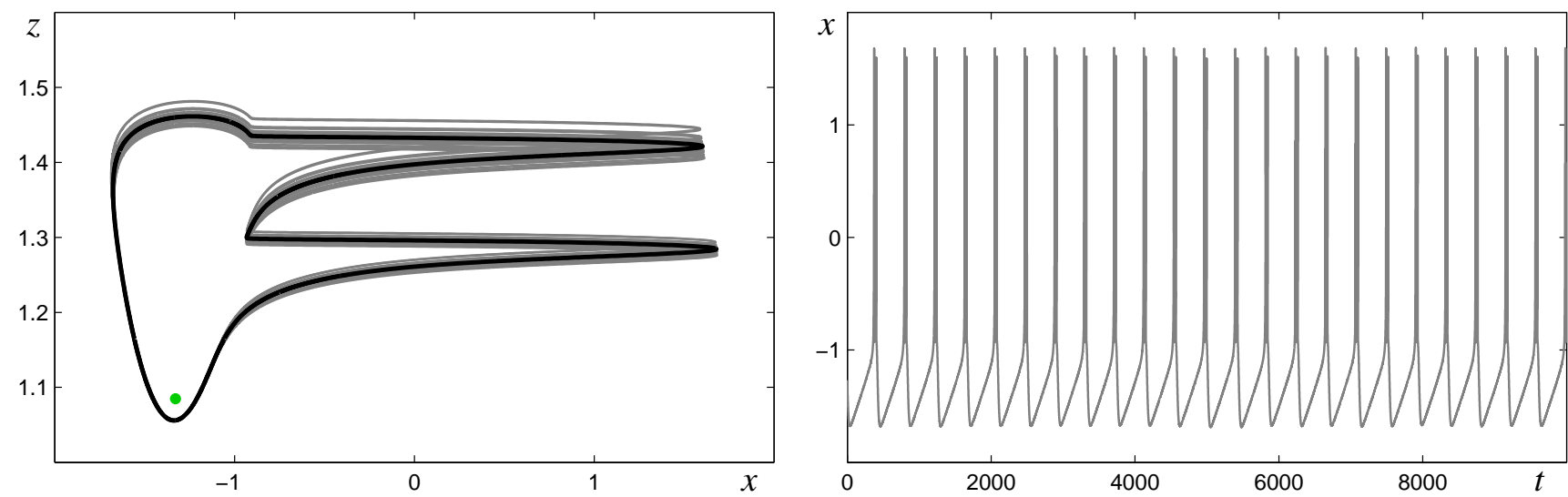

b)
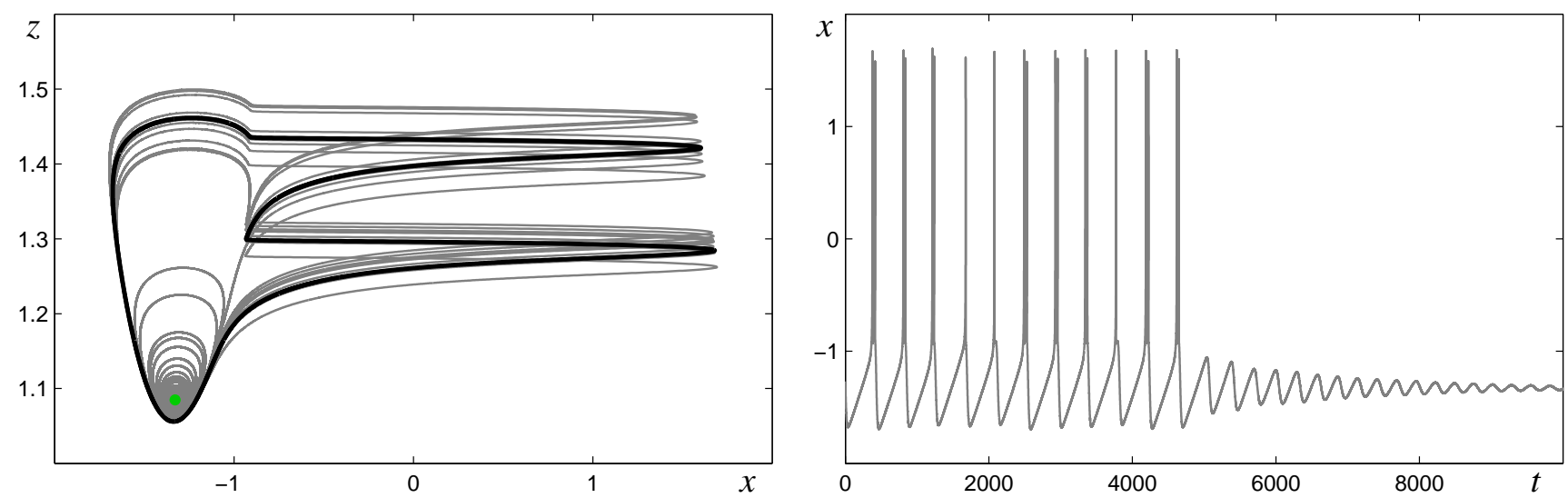

c)
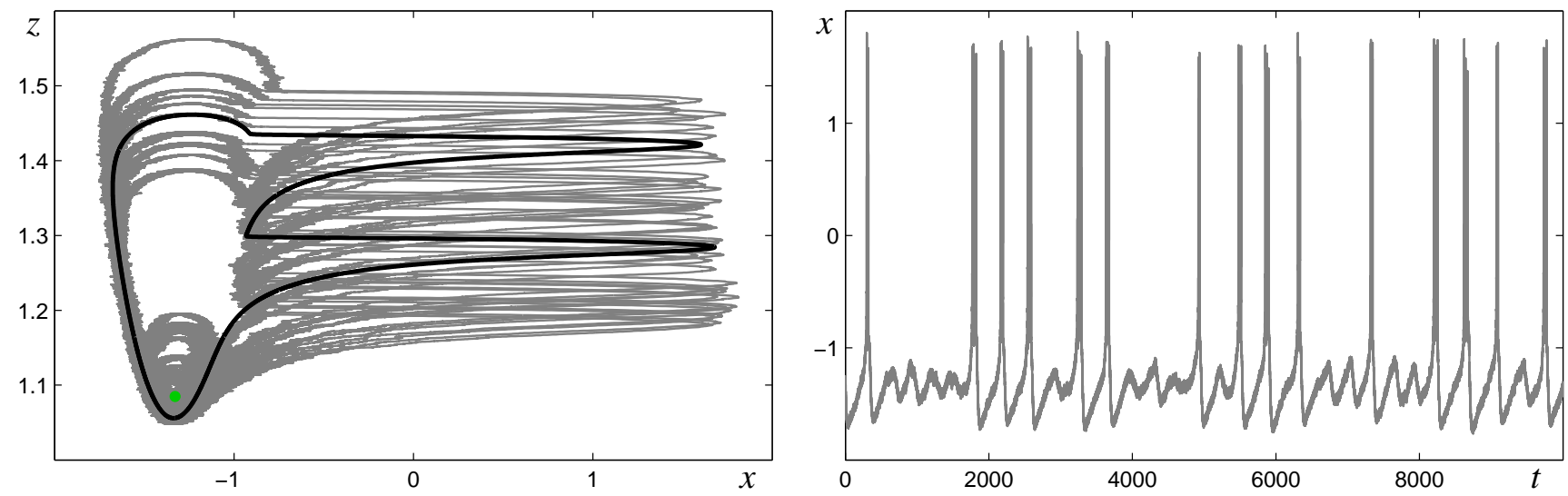

Fig. 10. Random trajectories starting from the limit cycle (in projection on $x O z$ plane) and corresponding time series for $I=1.27$ : a) $\varepsilon=0.0005$, b) $\varepsilon=0.002$, c) $\varepsilon=0.07$. For small noise (a), random trajectories are concentrated near the deterministic cycle. For a noise intensity value greater than some threshold (b), stochastic trajectories cross the separatrix, and go to the basin of attraction of the stable equilibrium. With a further increase of the noise intensity (c), backward transitions from the basin of attraction of the equilibrium to the basin of attraction of the limit cycle occur.

induced transition from the cycle to the equilibrium. Using Fig. 11, we can estimate empirically the critical values of the noise intensity of the onset of the noise-induced transition from the cycle to the equilibrium. For $I=1.27$, we get $\varepsilon^{*} \approx 0.001$ and for $I=1.28$ we have $\varepsilon^{*} \approx 0.009$.

Fig. 12 demonstrates changes of the probability density distribution of random trajectories. The probability density function $P(z)$ of $z$-coordinates of points of intersection of random trajectories with $x=\bar{x}$ is 
a)

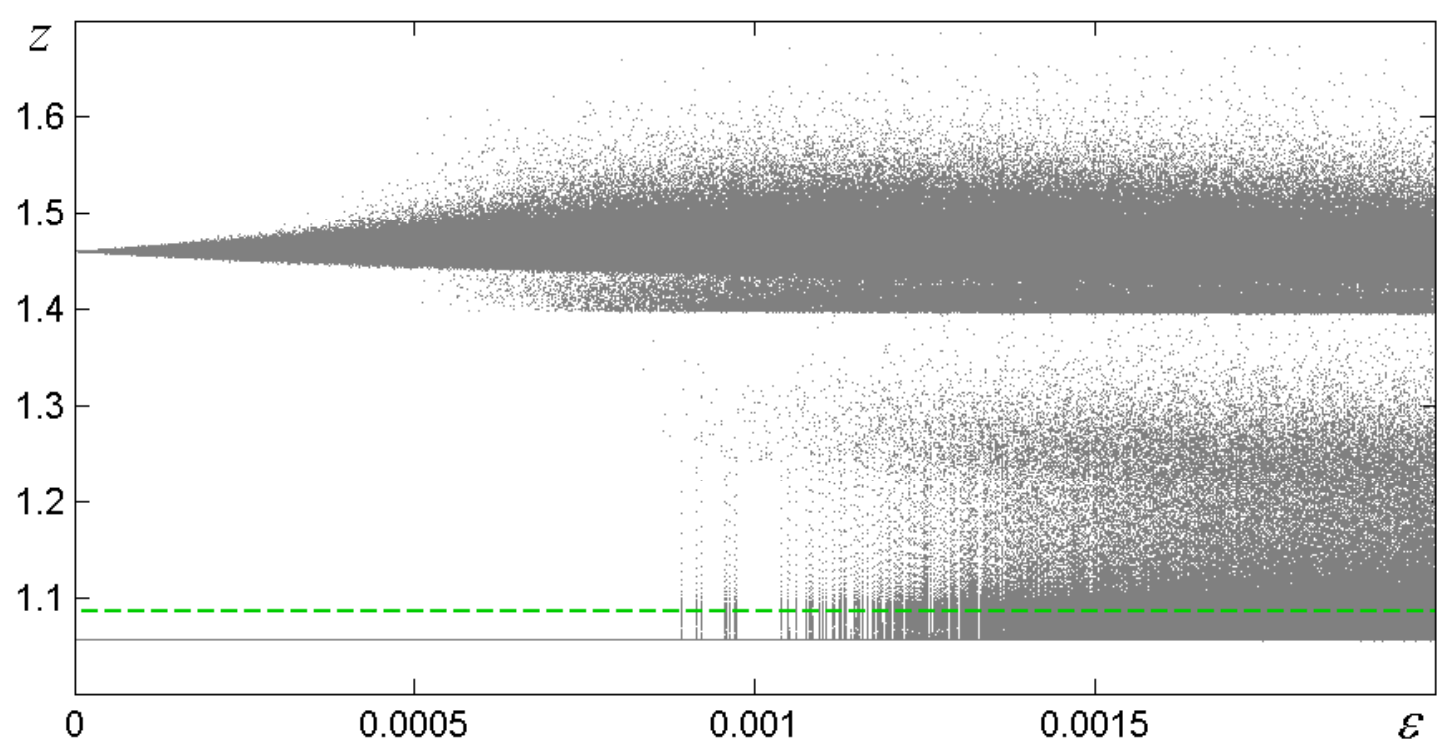

b)

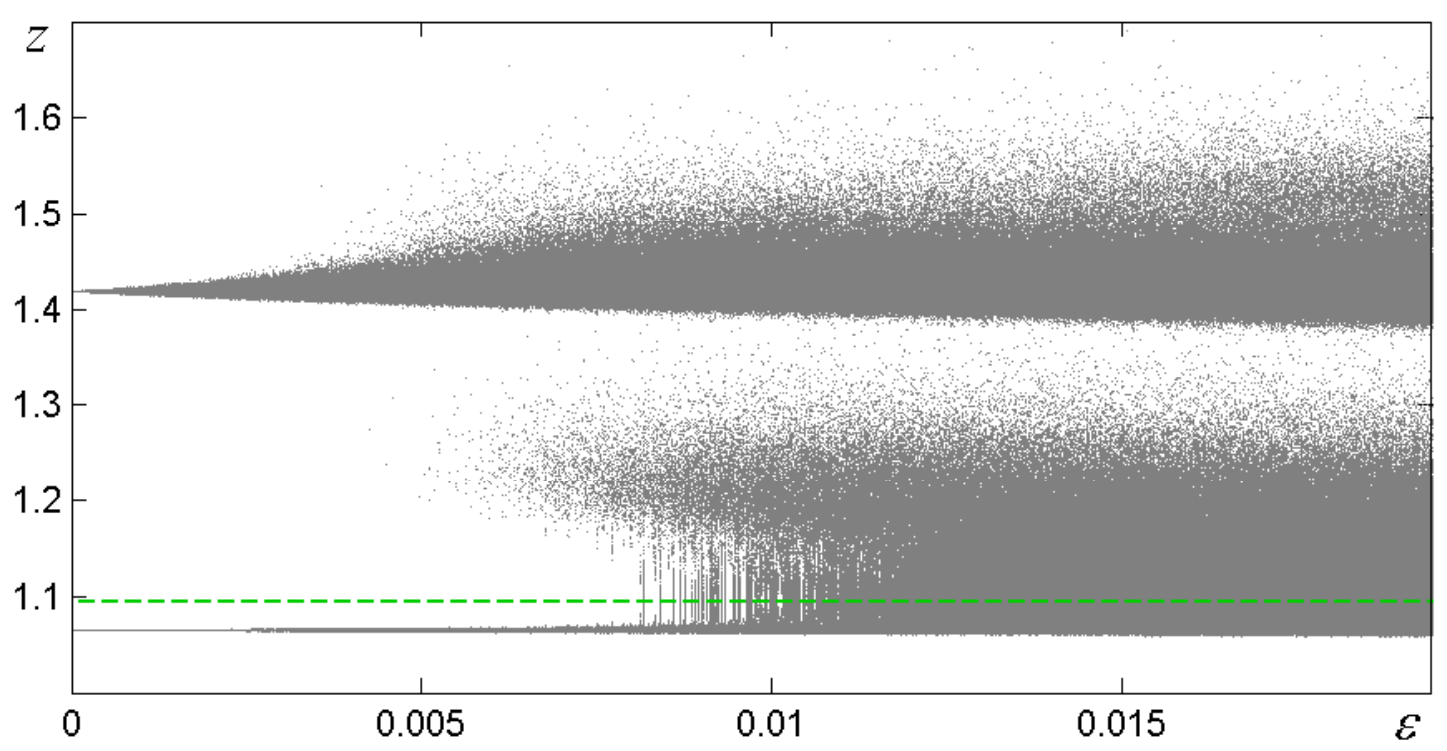

Fig. 11. Stochastic generation of bursting oscillations: $z$-coordinates of points of intersection of random trajectories with $x=\bar{x}$ in dependence on noise intensity $\varepsilon$ and $z$-coordinate of the equilibrium (dashed), a) $I=1.27$, b) $I=1.28$. For small noise intensities, random states concentrate near the deterministic limit cycle. With an increase of noise, random states near the equilibrium appear, corresponding to the onset of the noise-induced transition from the cycle to the equilibrium.

plotted for $I=1.27$ (Fig. 12a) and $I=1.28$ (Fig. 12b) for various noise intensities. For a weak noise, the probability density function is bimodal with peaks located above the limit cycle. With an increase in the noise intensity, the dispersion of random trajectories in the upper part of cycle increases. It is displayed in the plot of $P(z)$ so that its right peak becomes smaller and wider. With a further increase of $\varepsilon$, one can observe the appearance of a peak above the equilibrium, so $P(z)$ function becomes trimodal. Such qualitative change of the form of the probability density function is specified as $P$-bifurcation.

This phenomenon of noise-induced transitions is confirmed by changes of the function $\eta(\varepsilon)$ (see Fig. 13). 
a)

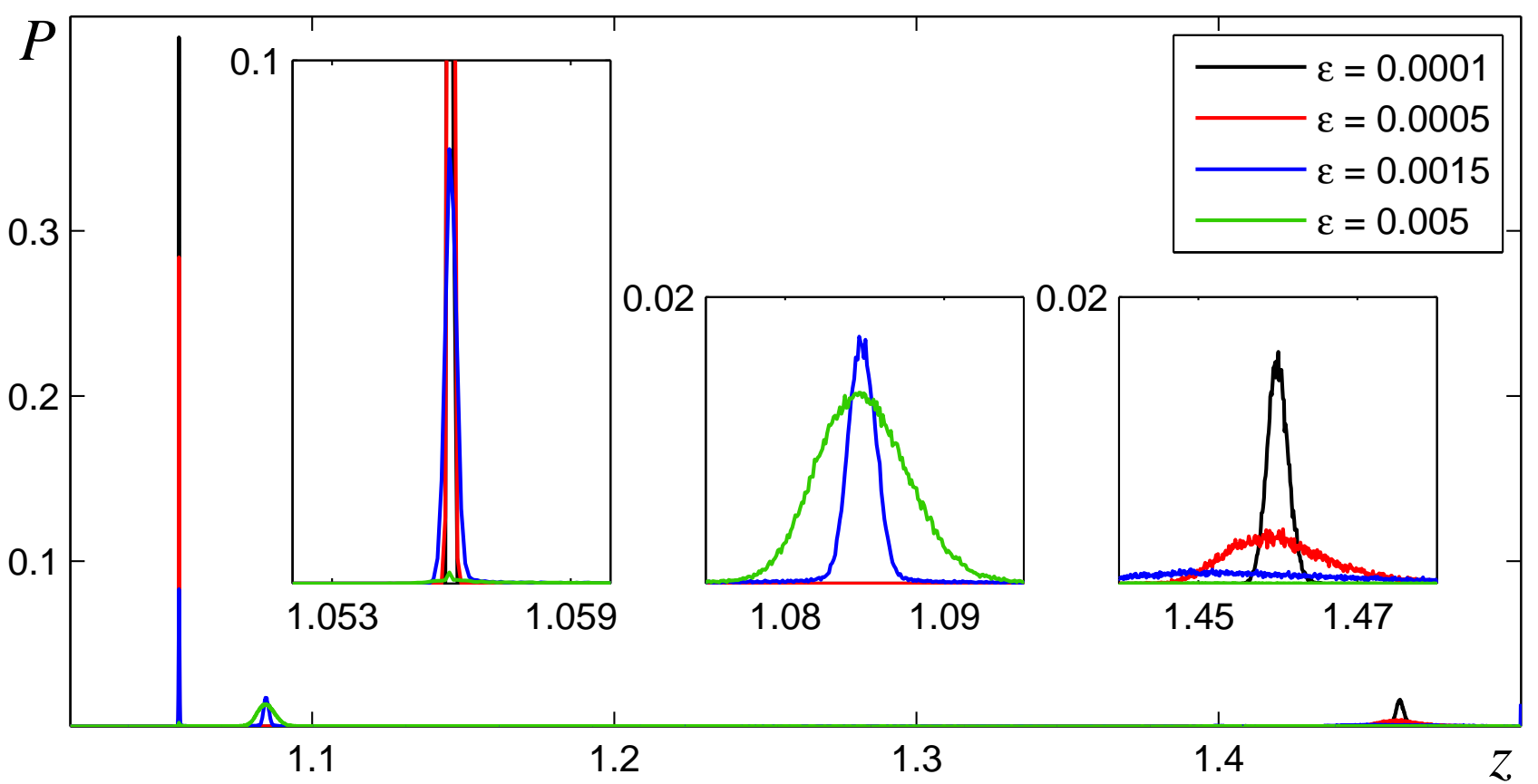

b)

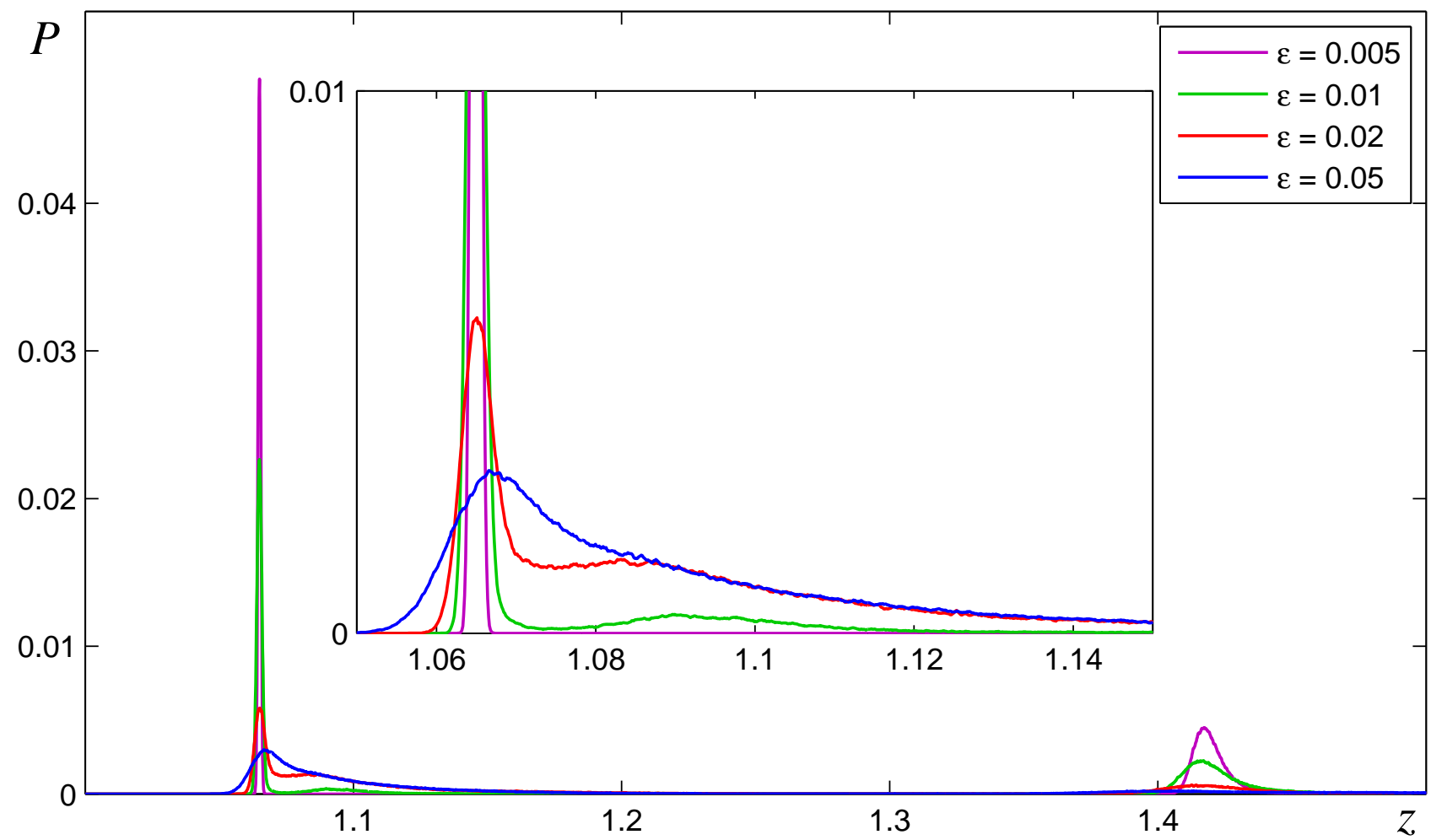

Fig. 12. Probability density distribution of random trajectories for a) $I=1.27$, b) $I=1.28$. For a weak noise, the probability density function is bimodal with peaks located above the limit cycle. With an increase of noise intensity, one can observe the appearance of a new peak above the equilibrium, and the function becomes trimodal. Thus, the system demonstrates $P$-bifurcation. 


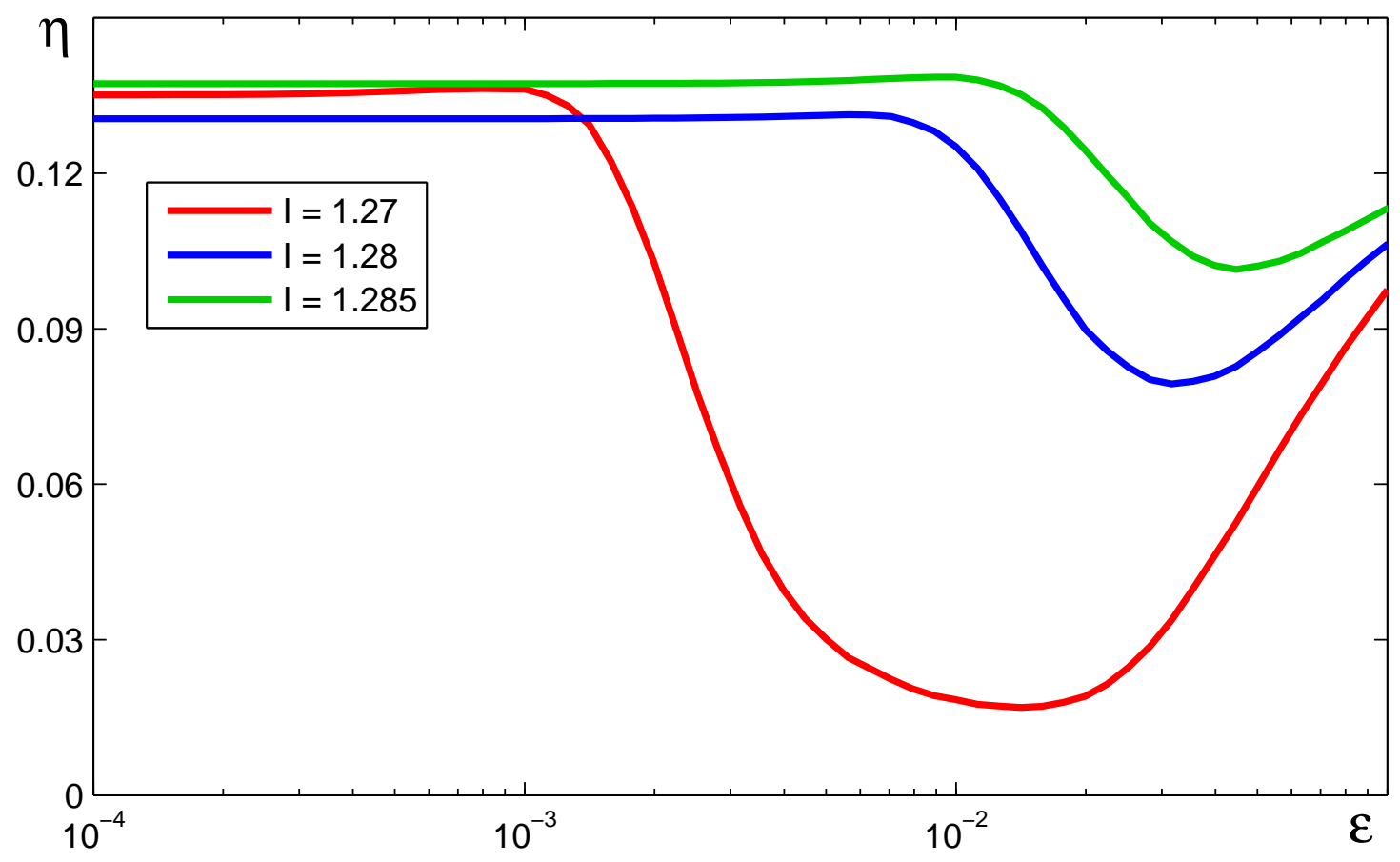

Fig. 13. Function $\eta=\frac{T_{l}}{T}$. Here, $T_{l}$ is the time spending by the system in the region $x>-1$ and $T$ is the total time. The value $x=-1$ is used as a threshold that separates in the phase space small-amplitude oscillations near equilibrium from the spiking phase. For a small noise, $\eta$ is almost constant. With the increase of noise, the decrease of $\eta$ is observed, which corresponds to the onset of noise-induced transitions from limit cycle to equilibrium. For greater noise intensity values, $\eta$ increases, which corresponds to the onset of backward transitions.

The function $\eta(\varepsilon)$ is almost constant for a small noise. For values of the noise intensity greater than some threshold, $\eta$ begins to decrease, which means that the system begins to spend less time in a vicinity of the limit cycle. For greater noise intensity values, $\eta$ increases, which corresponds to the onset of backward transitions. As one can observe, the threshold values of noise intensity found above $\left(\varepsilon^{*} \approx 0.001\right.$ for $I=1.27$, and $\varepsilon^{*} \approx 0.009$ for $I=1.28$ ), correspond to the onset of decrease of function $\eta$.

Let us study this effect using the SSF technique.

The non-zero eigenvalues of the SSF matrix of the limit cycle for $I=1.27$ are shown on Fig. 14. One can observe a significant overfall of values along the cycle. Note that the part of the cycle with minimal $x$-coordinates has the maximal stochastic sensitivity (maximal values of the largest eigenvalue). Other two peaks of SSF correspond to spikes in the cycle.

Fig. 14c shows a limit cycle with a stochastic trajectory for $I=1.27$ and $\varepsilon=0.003$. The trajectory goes to the basin of attraction of the equilibrium from the lower-right part of the cycle (shown as "transition region" in Fig. 14c).

Eigenvectors $v_{1}(t)$ corresponding to the largest eigenvalues $\lambda_{1}(t)$ of SSF localize the main directions for deviations of random trajectories from the cycle. Consider points $\xi_{i}$ of the limit cycle in the transition region and vectors of the main directions $v_{i}$ for each of these points. Fig. 15 shows the limit cycle for $I=1.28$ and lines passing through the points $\xi_{i}$ in the directions of $v_{i}$. Consider intervals starting in the points $\xi_{i}$ and ending on the separatrix surface, separating basins of attraction of the limit cycle and the equilibrium. Using these intervals we can estimate the critical value of the noise intensity corresponding to the onset of noise-induced transition from the cycle to equilibrium. For $I=1.27$ we get $\varepsilon^{*} \approx 0.0015$, for $I=1.275$ we have $\varepsilon^{*} \approx 0.0067$ and for $I=1.28$ we get $\varepsilon^{*} \approx 0.02$. The obtained values are in a good agreement with the results of the numerical simulations.

Using the approach described in Section 2.1, we estimate the critical values of the noise intensity for 
a)

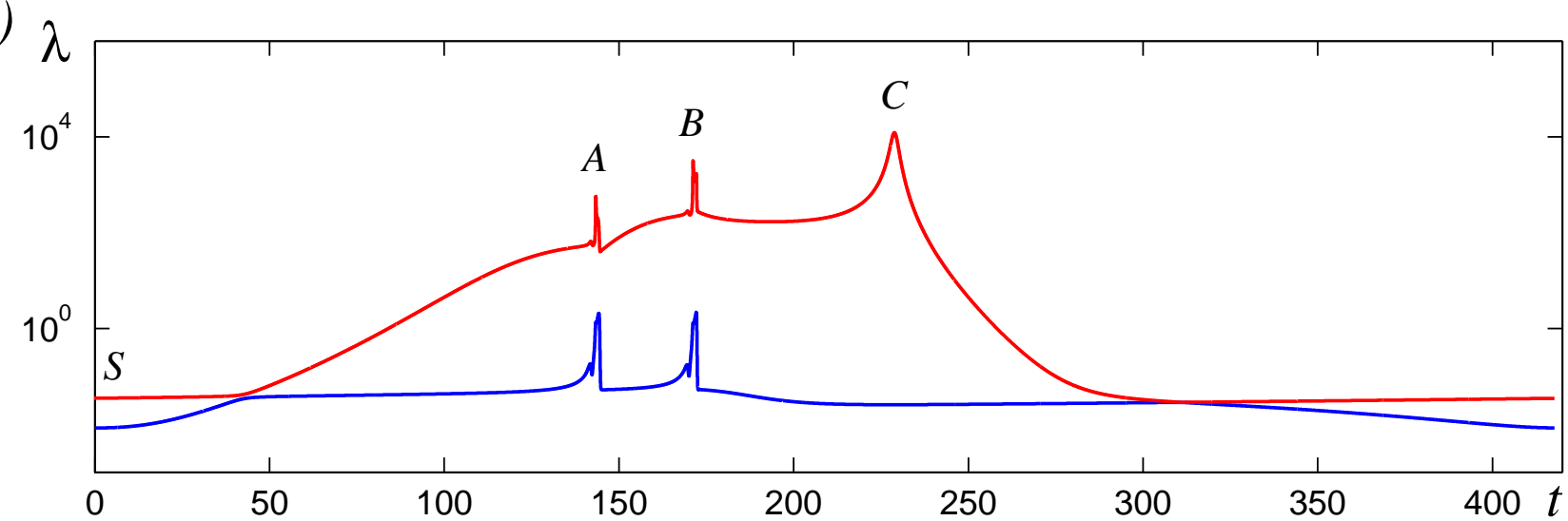

b)

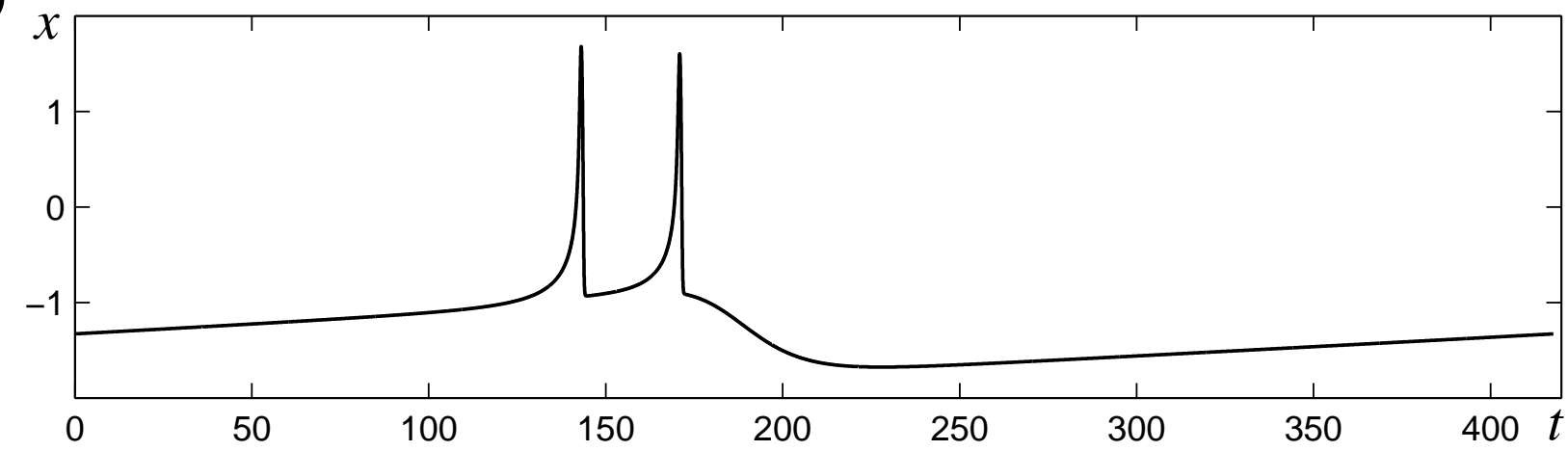

c)

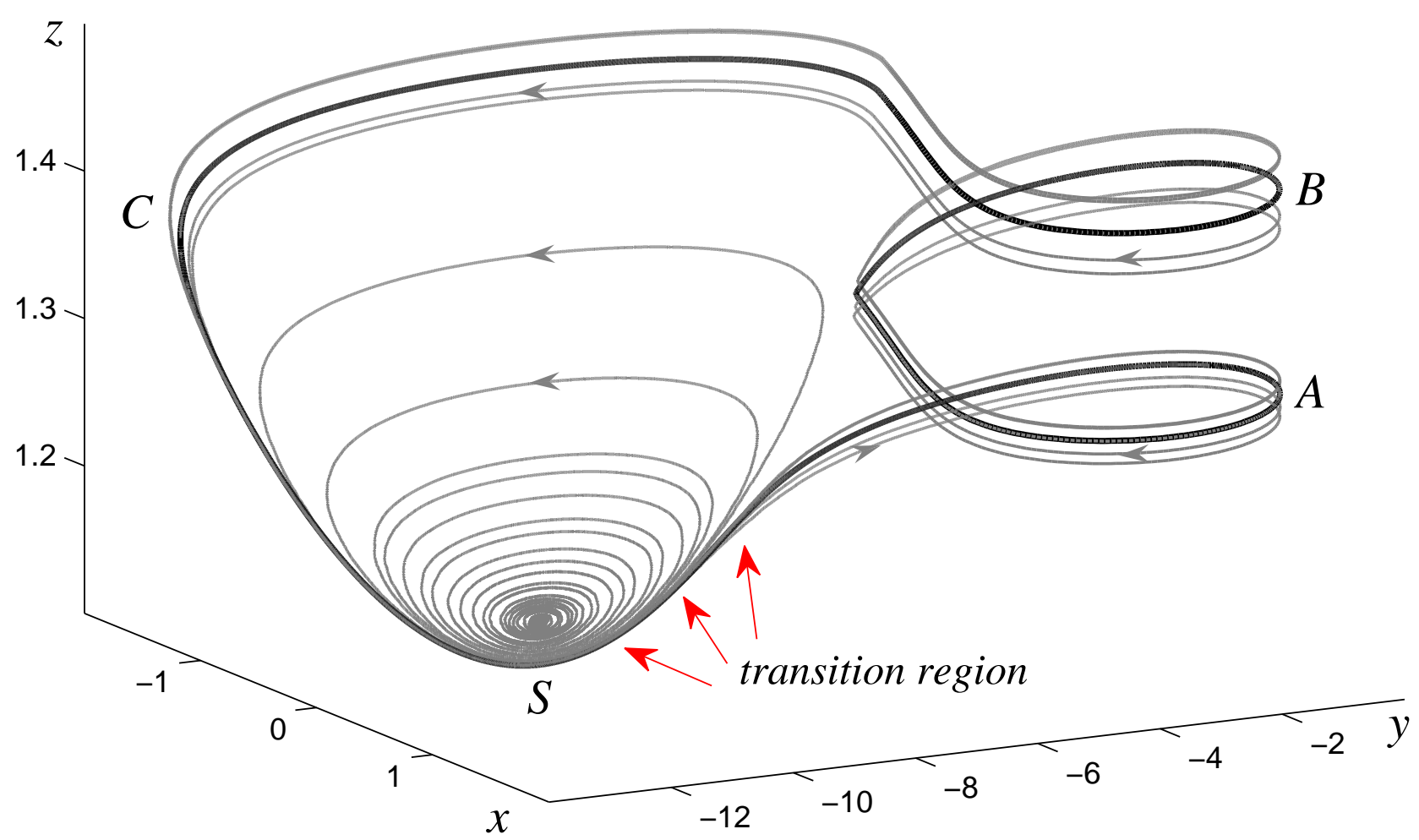

Fig. 14. a) Eigenvalues of the SSF for the limit cycle; b) $x$-coordinates of limit cycle for $I=1.27$; c) limit cycle (black) and stochastic trajectory (grey) for $I=1.27$ and $\varepsilon=0.003$. Peaks of the SSF correspond to the parts of the limit cycle with the highest stochastic sensitivity $(a, b)$. The trajectory goes to the basin of attraction of the equilibrium from the part of the cycle, shown as "transition region" in (c). 


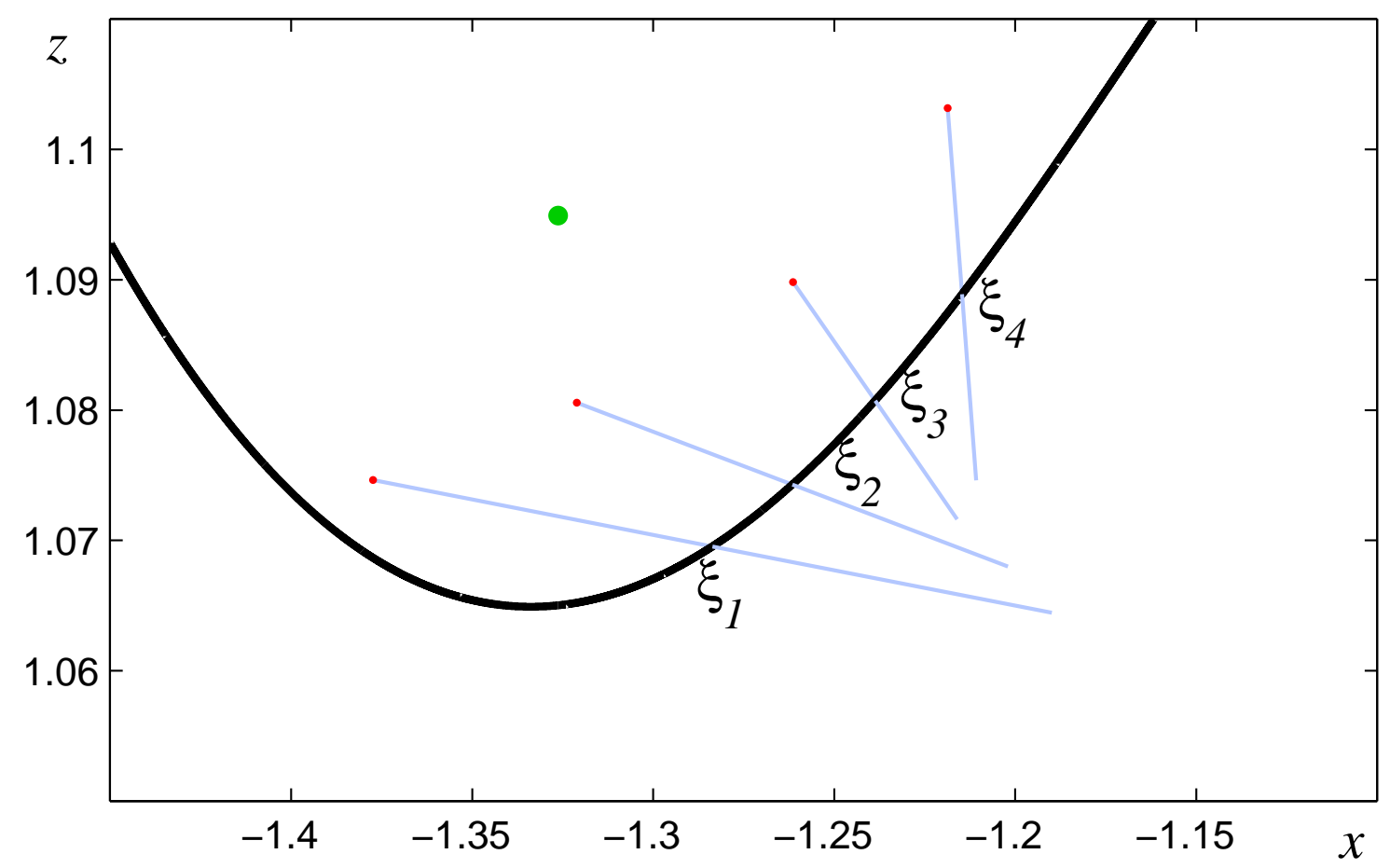

Fig. 15. Method of the main directions: limit cycle (solid black), equilibrium (green), directions of the eigenvectors corresponding to the largest eigenvalue of SSF (blue), points of the intersection with the separatrix (red) for $I=1.28$ (in the projection on $x O z)$.

the transitions from the equilibrium to the limit cycle. For $I=1.27$ we get $\varepsilon^{*} \approx 0.0254$, for $I=1.275$ we have $\varepsilon^{*} \approx 0.0205$ and for $I=1.28$ we get $\varepsilon^{*} \approx 0.0147$.

Let us compare the critical values of the noise intensity for the transition from cycle to equilibrium and vice versa. With the approaching of the parameter $I$ to the bifurcation point $I_{1}$, the equilibrium loses its stability, while the stability of the limit cycle grows. Fig. 16 shows that with the approaching of the parameter $I$ to the bifurcation point $I_{1}$, the critical values of the noise intensity for the equilibrium decrease, but the critical values for the cycle increase. This explains the fact that for the smaller values of $I$, the equilibrium dominates in the system, but closer to $I_{1}$, the limit cycle begins to dominate.

Fig. 17 shows plots of the function $\eta$ calculated over trajectories starting from the limit cycle, and starting from the equilibrium. For a weak noise, the value of $\eta$ is zero for the trajectories starting from the equilibrium and almost constant at non-zero rate for the limit cycle. The noise intensity value, for which $\eta$ for cycle begins to decrease, is the critical value $\varepsilon_{l c}^{*}$, corresponding to the onset of the transition from the limit cycle to the equilibrium. Similarly, the value $\varepsilon_{e q}^{*}$ is a critical value, corresponding to the onset of the transition from the equilibrium to the limit cycle: it is a noise intensity, for which $\eta$ for the equilibrium begins to increase. Values of noise intensity, for which the plots of $\eta$ for the cycle and the equilibrium begin to coalesce, correspond to the onset of the mutual transitions between the cycle and the equilibrium. For $I=1.27 \varepsilon_{l c}^{*}<\varepsilon_{e q}^{*}$, so the transitions from the cycle to the equilibrium occur for smaller noise intensity values than from the equilibrium to the cycle, and the equilibrium dominates in the system. For $I=1.285$ $\varepsilon_{e q}^{*}<\varepsilon_{l c}^{*}$, so the transitions from the equilibrium to the cycle occur for smaller noise intensity values, and the cycle dominates in the system.

\subsection{Average number of spikes in burst of the stochastic system}

The phenomena of the stochastic generation of large-amplitude oscillations and the noise-induced transitions are accompanied with changes of number of spikes in a burst. Fig. 18 represents the distribution for 


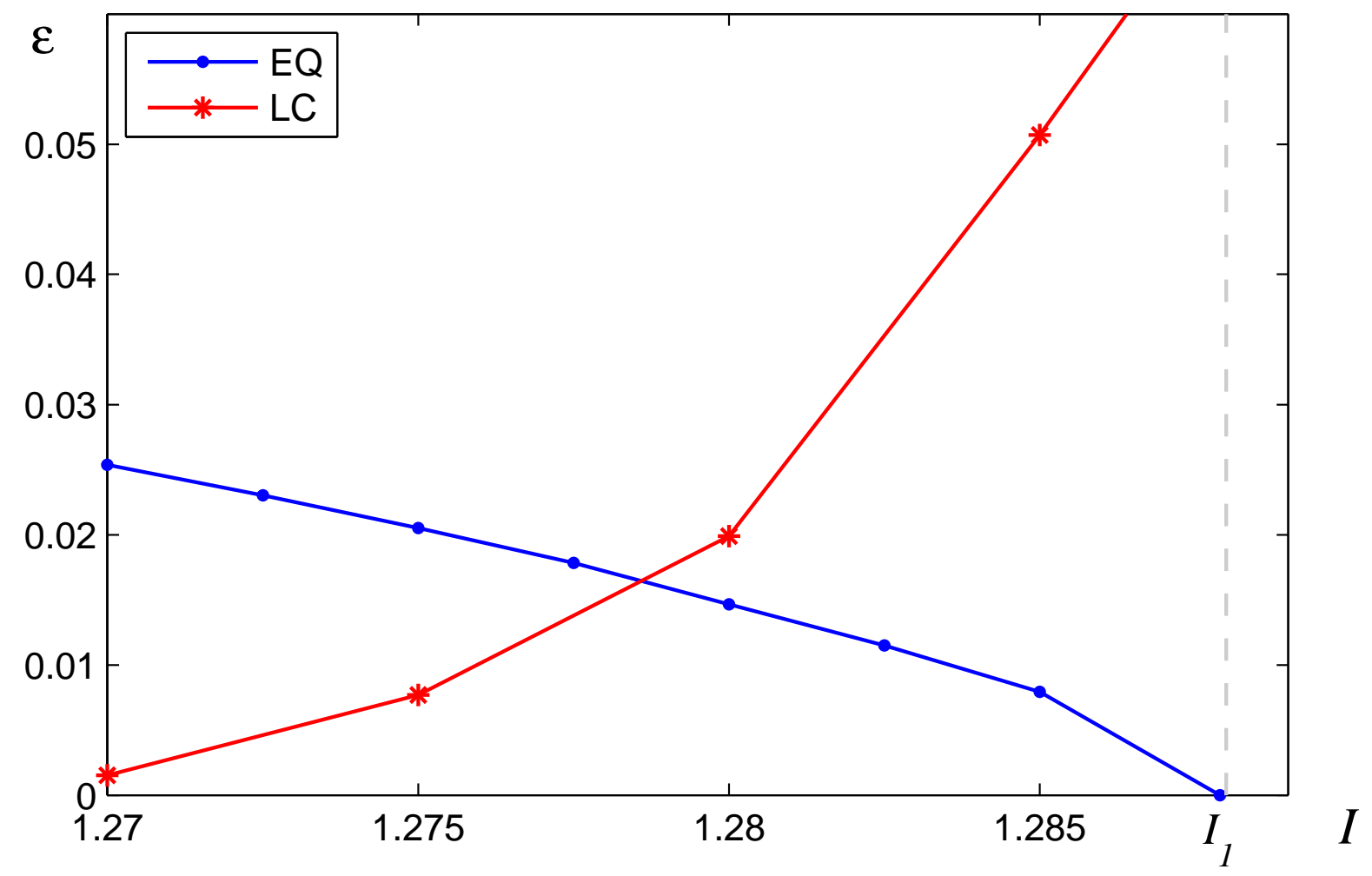

Fig. 16. Critical values of noise intensity for the transition from cycle to equilibrium and vice versa.

the number of spikes $n$ in bursts for $I=1.2, I=1.28, I=1.4, I=1.8$ and various $\varepsilon$.

For $I=1.2$, an equilibrium is the only attractor of the deterministic system. Under stochastic disturbances, bursting oscillations appear in the system. A number of spikes in a burst is random. Fig. 18a shows that with the increase in noise intensity, the average number of spikes in bursts grows. Note that for a low noise, the average number of spikes is two. It can be explained by the fact that with an increase in the noise intensity, trajectories with one and two spikes appear almost simultaneously, but trajectories with two spikes dominate.

In parametric zones where the system exhibits a limit cycle, the number of spikes in bursts also changes (see Figs. 18 b-d). Note that with an increase in the noise intensity, along with the dominating number of spikes, bursts with lower and greater numbers of spikes in bursts appear. Meanwhile, the average number of spikes increases. Fig. 19 summarizes these observations, showing the average number of spikes in bursts in the dependence on the noise intensity for the considered values of the parameter $I$.

The emergence of bursts with different numbers of spikes can be confirmed by probability density functions of interspike intervals (ISI). Note that the long ISIs correspond to intervals between the last spike in a burst and the next spike. The short ISIs correspond to intervals between spikes in burst. Fig. 20 shows the probability density functions of interspike intervals for $I=1.8$ (burst with 4 spikes). For a low noise, the functions have three peaks corresponding to short ISIs and one peak corresponding to long ISIs. With an increase in the noise intensity, a new peak corresponding to short ISIs appears.

\section{Noise-induced transitions from order to chaos}

It is well known that the dynamic peculiarities of stochastic flows can be quantitatively described by Lyapunov exponents. A negativeness of the largest Lyapunov exponent (LLE) means that the trajectories of the stochastic system mostly converge. Positiveness of LLE indicates that in the stochastic flow the divergence dominates. Change of the sign of LLE from negative to positive ( $D$-bifurcation) is a standard 
a)

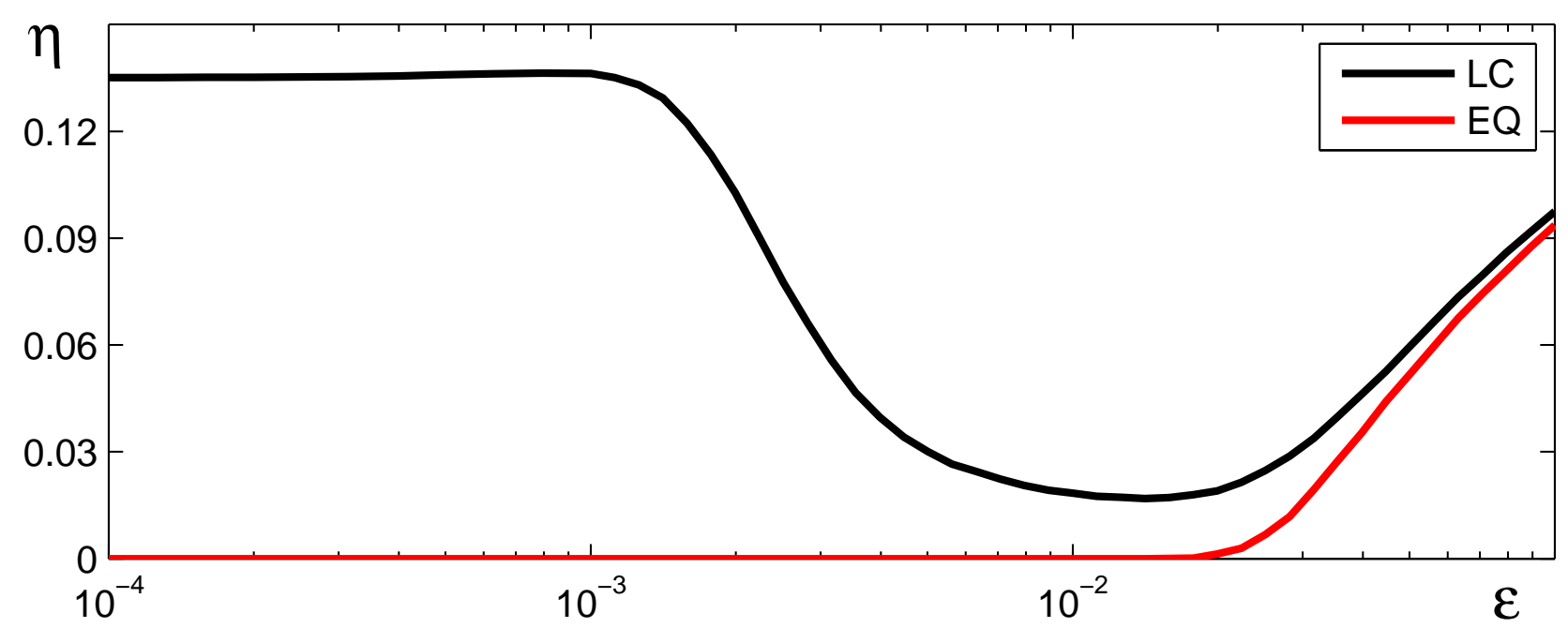

b)

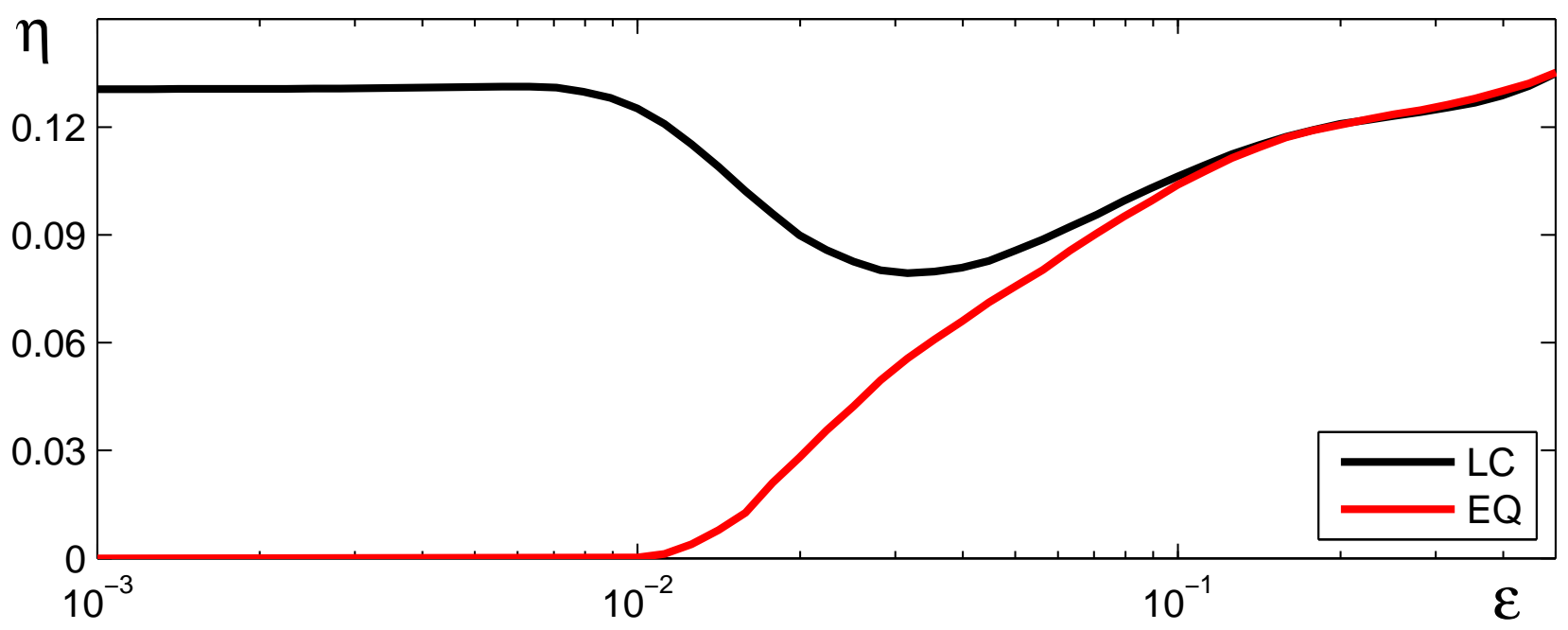

c)

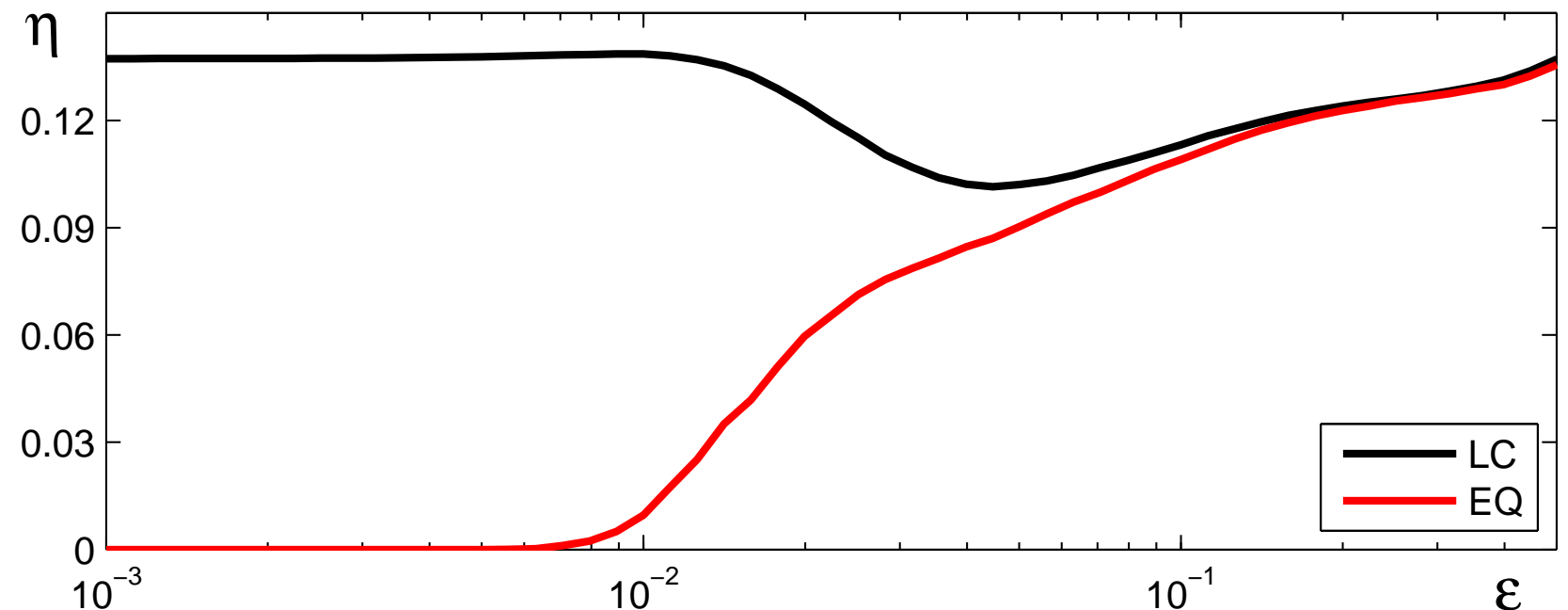

Fig. 17. Function $\eta$ calculated over trajectories starting from the limit cycle (black) and the equilibrium (red): a) $I=1.27$, b) $I=1.28$ c) $I=1.285$. 
a)

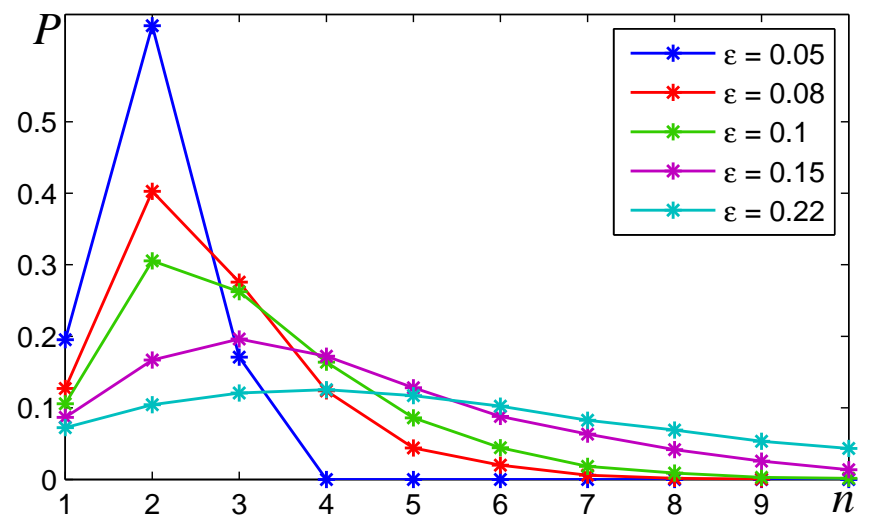

c)

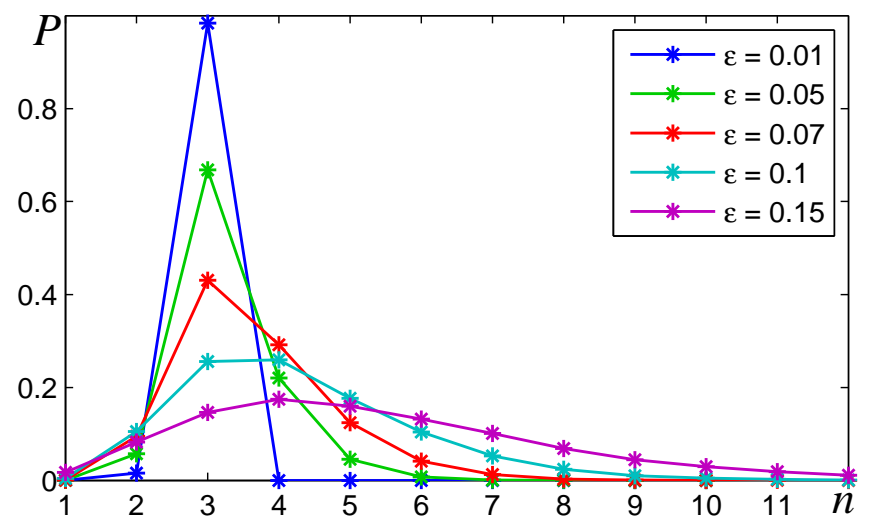

b)

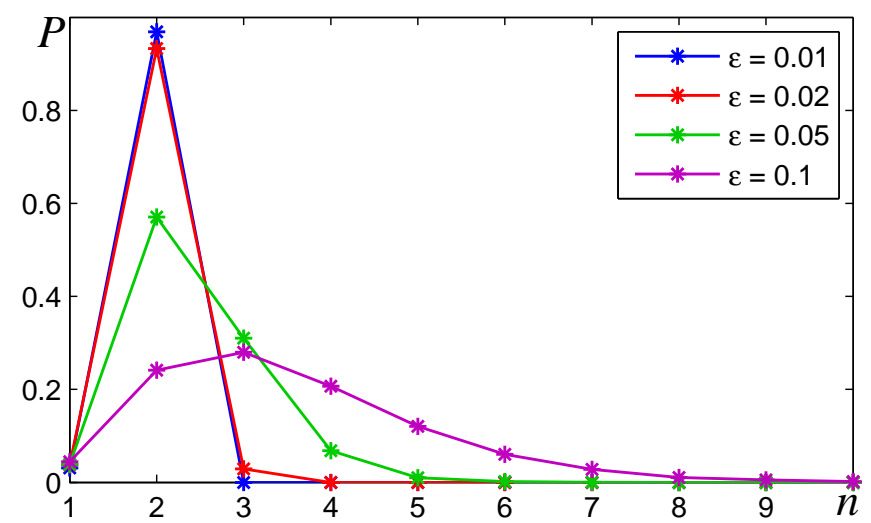

d)

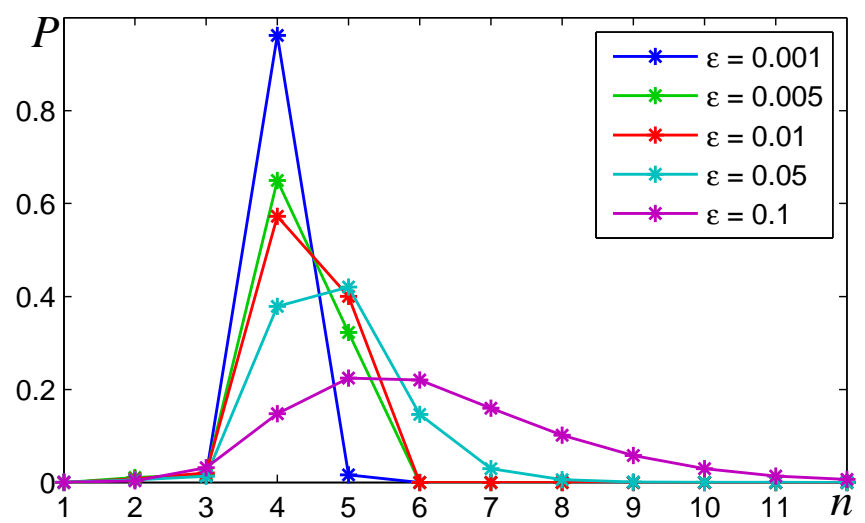

Fig. 18. Distribution for number of spikes in burst for a) $I=1.2$ b) $I=1.28$ c) $I=1.4$ d) $I=1.8$.

justification of the transition from regular to chaotic dynamics [Matsumoto \& Tsuda, 1983; Gassmann, 1997; Gao et al., 1999].

Let us discuss how noise-induced phenomena of bursting generation in system (2) relate to the variation of the LLE. First consider the zone $I<I_{0}$ of the monostability, where the stable equilibrium is a single attractor of the deterministic system. In Fig. 21a, plots of $\Lambda(\varepsilon)$ are shown for $I=1.2$ and $I=1.25$. Here, LLE was calculated by standard Benettin method over the trajectories starting from the deterministic equilibrium.

For a weak noise, the function $\Lambda(\varepsilon)$ has negative values and practically does not change with the noise increasing. This means that dynamics of stochastic flows is regular, with small-amplitude random oscillations (see Figs. 3,4). As the noise intensity increases, the function $\Lambda(\varepsilon)$ changes its behavior. LLE begins to increase and changes the sign from minus to plus, indicating the transition from order to chaos. Values of the noise intensity corresponding to such change of the sign essentially depend on the parameter $I$. The higher the value of $I$, the lower the noise transforming the system from order to chaos. So, comparing the Fig. 4 and Fig. 21a, we can resume that the noise-induced generation of bursts is accompanied by the chaotization of the stochastic flows.

Further consider the zone of bistability $I_{0}<I<I_{1}$ where a stable equilibrium and a stable limit cycle coexist. In Fig. 21b, plots of $\Lambda(\varepsilon)$ for $I=1.27, I=1.28$ are shown. Here, LLE was calculated over the trajectories starting from the deterministic limit cycle. Note that evidently $\Lambda(0)=0$.

Here, for a weak noise, functions $\Lambda(\varepsilon)$ decrease. The negativeness of LLE reflects a noise-induced stabilization of stochastic flows. As the noise intensity increases, the function $\Lambda(\varepsilon)$ begins to grow, changes its sign, and becomes positive. So, increasing noise transforms the behavior of this system from regular to chaotic in the bistability zone as well.

Thus, one can conclude that noise-induced generation of bursts is accompanied by $D$-bifurcations with 


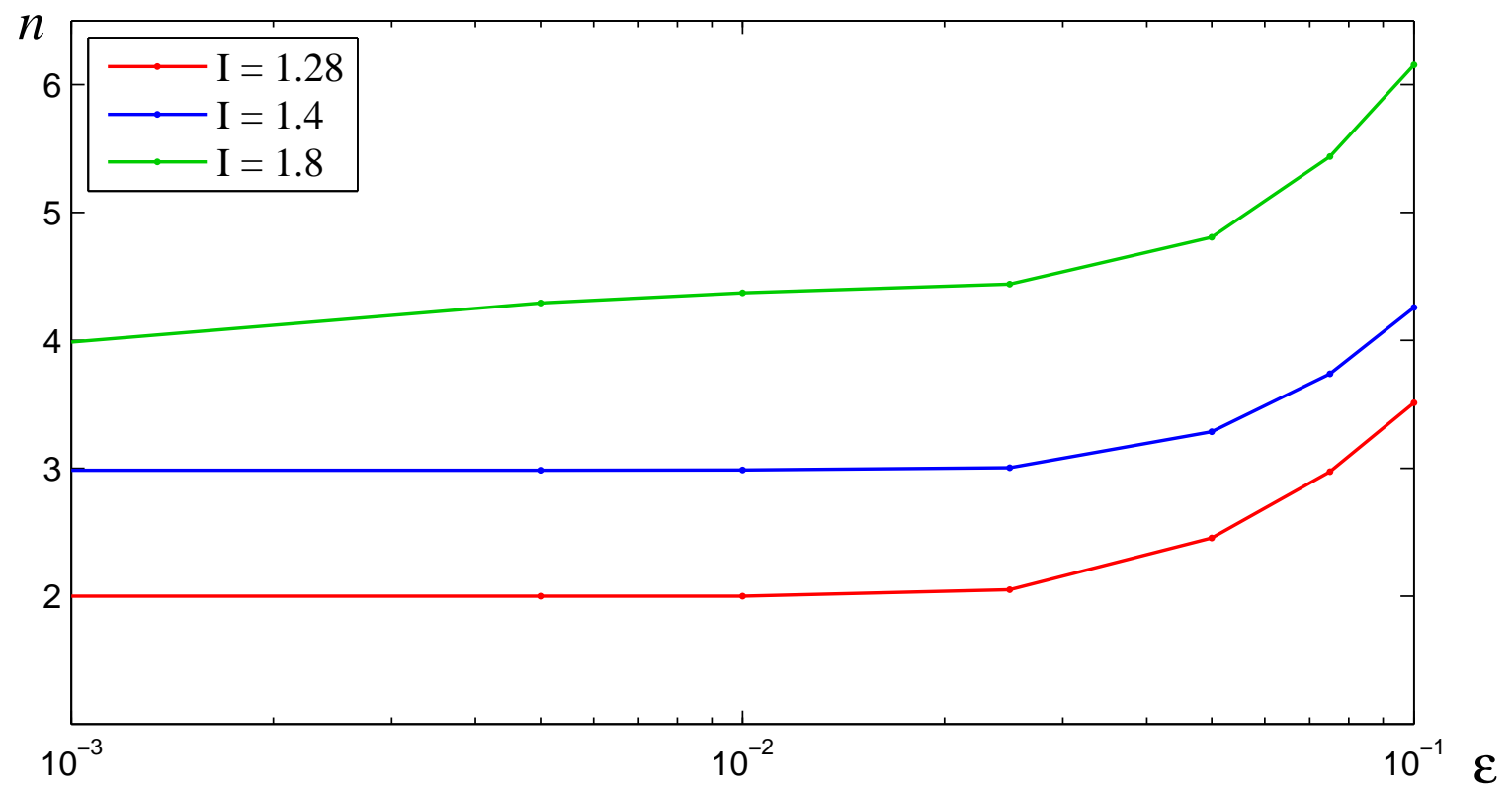

Fig. 19. Average number of spikes in burst versus noise intensity.

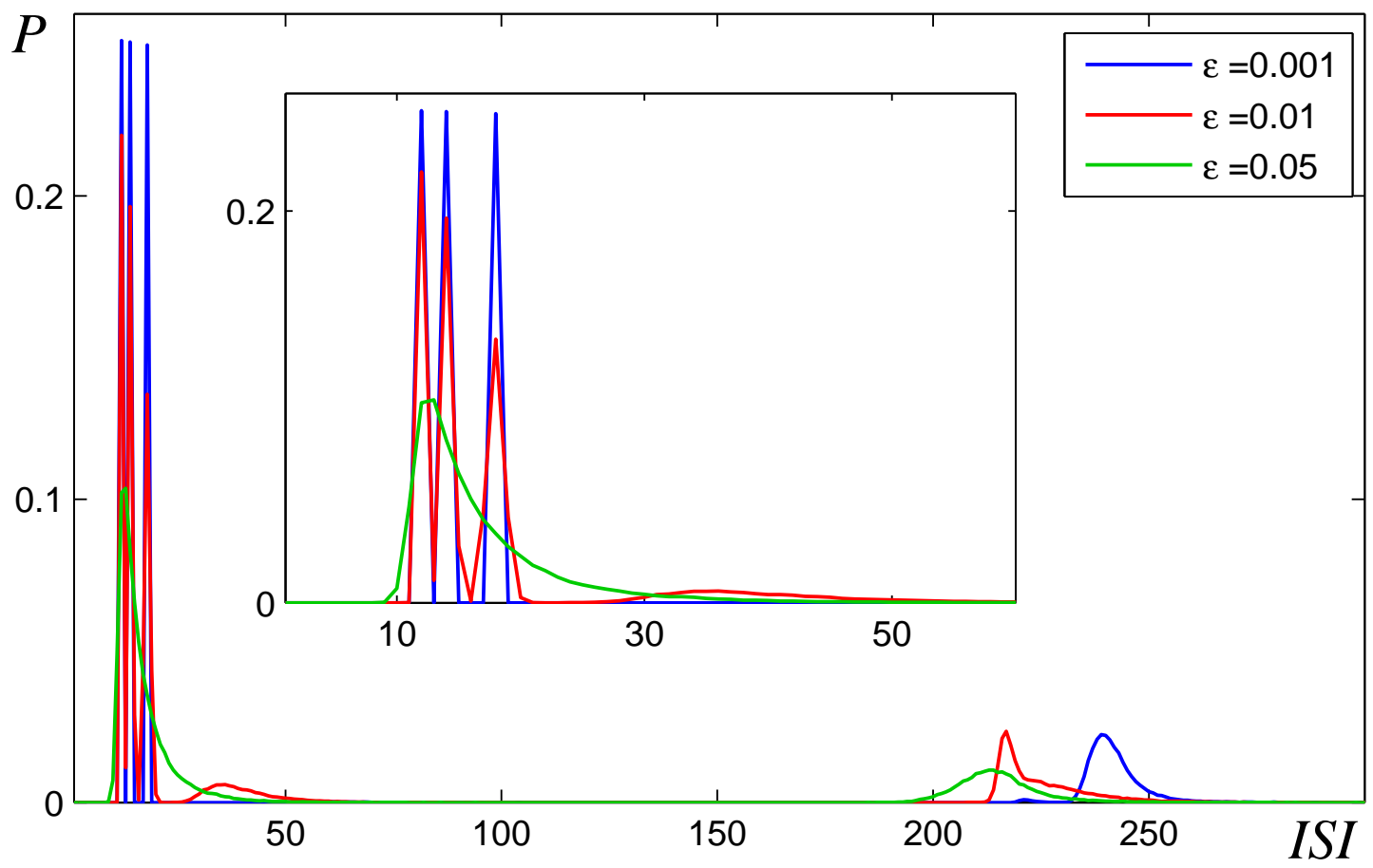

Fig. 20. Probability density functions of interspike intervals (ISIs) for $I=1.8$ (burst with 4 spikes). Peaks in the zone of short ISIs correspond to spiking phase in burst, and a peak in the zone of long ISIs corresponds to the quiescence phase. With an increase in the noise intensity, a new peak in the zone of short ISIs appears.

transitions to chaos for both monostability and bistability zones. 


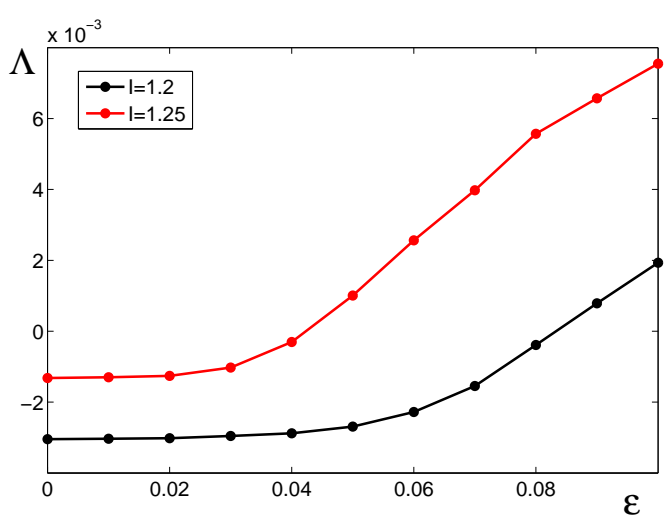

a)

Fig. 21. Largest Lyapunov exponents. Noise-induced generation of bursts is accompanied by D-bifurcations with transitions to chaos for both monostability and bistability zones.

\section{Conclusion}

The effect of random disturbances on the 3D Hindmarsh-Rose model was studied. We considered two parametric zones: monostability zone, where a stable equilibrium is the single attractor in the deterministic system, and bistability zone, where the deterministic dynamics is characterized by the coexistence of a stable equilibrium and a limit cycle. We have shown that in both cases, under the noise, a bursting-like behavior is observed. In the monostable zone, this phenomenon is connected with the stochastic generation of largeamplitude oscillations due to high excitability of the system. In the bistable zone, bursts appear due to the noise-induced transitions between attractors. We have suggested an approach for quantitative analysis of such stochastic $P$-bifurcations based on the stochastic sensitivity technique and the method of confidence domains. A number of spikes in noise-induced bursts is random. We have provided the probabilistic analysis of these numbers. We have shown that the noise-induced generation of bursts is related to $D$-bifurcations with transitions from order to chaos.

\section{Acknowledgments}

The analysis of the deterministic HR model was carried out by S.F. The work on the stochastic analysis was carried out by I.B., L.R., E.S., and supported by Russian Science Foundation (N 16-11-10098).

\section{Appendix A Stochastic sensitivity function technique}

Consider a nonlinear system of stochastic differential equations:

$$
d x=f(x) d t+\varepsilon \sigma(x) d w(t) .
$$

Here, $x$ is $n$-vector function, $w(t)$ is $n$-dimensional standard Wiener process, $\varepsilon$ is a scalar parameter of noise intensity.

Under stochastic disturbances, random trajectories of the system (A.1) leave the deterministic attractor (cycle or equilibrium) and form some probabilistic distribution around it. Full description of this distribution is given by Kolmogorov-Fokker-Planck (KFP) equation. In a steady regime, one can consider stationary probability density function $\rho(x, \varepsilon)$ governed by the stationary KFP equation.

To avoid well-known technical difficulties with the direct use of this equation, various asymptotics and approximations are developed [Kurrer \& Schulten, 1991; Lindner \& Schimansky-Geier, 1999; Milshtein \& Ryashko, 1995]. For the approximation of KFP solutions, a well-known quasipotential method [Freidlin \& Wentzell, 1984; Dembo \& Zeitouni, 1995] and a stochastic sensitivity function (SSF) technique [Bashkirtseva \& Ryashko, 2005, 2004, 2011] can be applied.

At first, let the system (A.1) have an exponentially stable equilibrium $\bar{x}$. The corresponding approximation of the quasipotential near $\bar{x}$ gives an asymptotic of the stationary distribution of random states of 
the system (A.1) in the Gaussian form:

$$
\rho(x, \varepsilon)=K \exp \left(-\frac{\left(x-\bar{x}, W^{-1}(x-\bar{x})\right)}{2 \varepsilon^{2}}\right) .
$$

For the exponentially stable equilibrium $\bar{x}$, the matrix $W$ is the unique solution of the equation

$$
F W+W F^{\top}=-S, \quad F=\frac{\partial f}{\partial x}(\bar{x}), \quad S=G G^{\top}, \quad G=\sigma(\bar{x}) .
$$

The matrix $W$ connects the stochastic input $\left(\varepsilon^{2}\right)$ and the stochastic output (a covariance matrix $D): \quad D(\varepsilon)=\varepsilon^{2} W$. So, the matrix $W$ can be considered as a coefficient of stochastic sensitivity of the system to random disturbances. We will call it the stochastic sensitivity matrix. This matrix characterizes a spatial arrangement of the stationary distributed random states of the stochastic system (A.1) around the deterministic equilibrium $\bar{x}$. Eigenvectors $v_{i}$ of the matrix $W$ determine a spatial arrangement of a confidence ellipsoid, whilst eigenvalues $\lambda_{i}$ define its size. In the special case when the coordinates of random states are uncorrelated and the variances in all directions are the same, confidence domains are spheres.

Consider confidence intervals in the direction of $v_{i}$. The ends of the confidence intervals are points of intersection of the confidence ellipsoid with a line passing in the direction of $v_{i}$. Their coordinates can be written as $x_{1,2}=\bar{x} \pm \alpha_{i} v_{i}$, where $\alpha_{i}=\sqrt{2 \lambda_{i}} \varepsilon k$, the coefficient $k$ is determined by the three-sigma rule $k=\frac{3}{\sqrt{2}}$. The confidence intervals allow to estimate critical values of noise intensity: $\varepsilon_{i}^{*}=\frac{\alpha_{i}^{*}}{\sqrt{2 \lambda_{i}} k}$.

Consider now a case when the corresponding deterministic system $(\varepsilon=0)$ has a $T$-periodic solution $x=\xi(t)$ with an exponentially stable phase curve $\Gamma$ (limit cycle). Let $\Pi_{t}$ be a hyperplane that is orthogonal to the cycle at the point $\xi(t)(0 \leq t<T)$. For this plane, in the neighborhood of the point $\xi(t)$, a Gaussian approximation of the stationary probabilistic distribution can be written [Bashkirtseva \& Ryashko, 2004] as:

$$
\rho_{t}(x, \varepsilon)=K \exp \left(-\frac{(x-\xi(t))^{\top} W^{+}(t)(x-\xi(t))}{2 \varepsilon^{2}}\right)
$$

with the mean value $m_{t}=\xi(t)$ and the covariance matrix $D(t, \varepsilon)=\varepsilon^{2} W(t)$. The stochastic sensitivity matrix $W(t)$ is a unique solution of the boundary problem

$$
\dot{W}=F(t) W+W F^{\top}(t)+P(t) S(t) P(t)
$$

with conditions

$$
W(T)=W(0), \quad W(t) r(t)=0 .
$$

Here

$$
\begin{gathered}
F(t)=\frac{\partial f}{\partial x}(\xi(t)), S(t)=G(t) G^{\top}(t), G(t)=\sigma(\xi(t)), \\
r(t)=f(\xi(t)), P(t)=P_{r(t)}, P_{r}=I-\frac{r r^{\top}}{r^{\top} r} .
\end{gathered}
$$

The eigenvalues $\lambda_{i}(t)$ and eigenvectors $v_{i}(t)$ of the SSF matrix characterize the distribution of random states in the Poincare section $\Pi_{t}$ near the point $\xi(t)$ of the cycle. SSF matrix allows to construct confidence ellipse with the center in point $\xi(t)$. A set of these ellipses for $t \in[0 ; T)$ specify some confidence torus around a deterministic cycle. This torus is a confidence domain in a phase space for the stochastic cycle as a whole [Ryashko et al., 2009].

Consider the point of cycle $\xi(t)$ and a confidence interval in the direction of $v_{i}(t)$. Ends of this confidence interval are points of intersection of the confidence torus with a line passing in the direction of $v_{i}(t)$. Their coordinates can be written as $x_{1,2}(t)=\xi(t) \pm \alpha_{i}(t) v_{i}(t)$, where $\alpha_{i}(t)=\sqrt{2 \lambda_{i}(t)} \varepsilon k$, the coefficient $k$ is determined by the three-sigma rule $k=\frac{3}{\sqrt{2}}$. The confidence intervals allow to estimate critical values of noise intensity: $\varepsilon_{i}^{*}=\min _{t \in[0 ; T)} \frac{\alpha_{i}^{*}(t)}{\sqrt{2 \lambda_{i}(t)} k}$. 


\section{References}

Anishchenko, V. S., Astakhov, V. V., Neiman, A. B., Vadivasova, T. E. \& Schimansky-Geier, L. [2007] Nonlinear Dynamics of Chaotic and Stochastic Systems. Tutorial and Modern Development (SpringerVerlag, Berlin, Heidelberg).

Arnold, L. [1998] Random Dynamical Systems (Springer-Verlag).

Baltanas, J. \& Casado, J. [2002] "Noise-induced resonances in the Hindmarsh-Rose neuronal model," Phys. Rev. E 65, 041915.

Barrio, R., Martinez, M. A., Serrano, S. \& Shilnikov, A. [2014] "Macro- and micro-chaotic structures in the Hindmarsh-Rose model of bursting neurons," Chaos 24, 023128.

Barrio, R. \& Shilnikov, A. [2011] "Parameter-sweeping techniques for temporal dynamics of neuronal systems: case study of Hindmarsh-Rose model," Journal of mathematical neuroscience 1, 6 .

Bashkirtseva, I. \& Ryashko, L. [2005] "Sensitivity and chaos control for the forced nonlinear oscillations," Chaos, Solitons and Fractals 26, 1437-1451.

Bashkirtseva, I. \& Ryashko, L. [2011] "Sensitivity analysis of stochastic attractors and noise-induced transitions for population model with Allee effect," Chaos 21, 047514.

Bashkirtseva, I. A. \& Ryashko, L. B. [2004] "Stochastic sensitivity of 3D-cycles," Mathematics and Computers in Simulation 66, 55-67.

Bashkirtseva, I., Ryashko, L. \& Slepukhina, E. [2014] "Noise-induced oscillation bistability and transition to chaos in FitzHugh-Nagumo model," Fluctuation and Noise Letters 13, 1450004.

Dembo, M. \& Zeitouni, O. [1995] Large deviations techniques and applications (Jones and Bartlett Publishers, Boston).

Desroches, M., Kaper, T. \& Krupa, M. [2013] "Mixed-mode bursting oscillations: Dynamics created by a slow passage through spike-adding canard explosion in a square-wave burster," Chaos 23, 046106.

Freidlin, M. I. \& Wentzell, A. D. [1984] Random Perturbations of Dynamical Systems (Springer, New York).

Gammaitoni, L., Hanggi, P., Jung, P. \& Marchesoni, F. [1998] "Stochastic resonance," Rev. Mod. Phys. 70, 223-287.

Gao, J. B., Hwang, S. K. \& Liu, J. M. [1999] "When can noise induce chaos?" Phys. Rev. Lett. 82, $1132-1135$.

Gassmann, F. [1997] "Noise-induced chaos-order transitions," Phys. Rev. E. 55, 2215-2221.

Gonzalez-Miranda, J. M. [2003] "Observation of a continuous interior crisis in the Hindmarsh-Rose neuron model," Chaos 13, 845-852.

Gu, H., Yang, M., Li, L., Liu, Z. \& Ren, W. [2002] "Experimental observation of the stochastic bursting caused by coherence resonance in a neural pacemaker," Neuroreport 13, 1657-1660.

Hindmarsh, J. L. \& Rose, R. M. [1984] "A model of neuronal bursting using three coupled first order differential equations," Proc R Soc Lond B Biol Sci 221, 87-102.

Horsthemke, W. \& Lefever, R. [1984] Noise-Induced Transitions (Springer, Berlin).

Innocenti, G., Morelli, A., Genesio, R. \& Torcini, A. [2007] "Dynamical phases of the Hindmarsh-Rose neuronal model: Studies of the transition from bursting to spiking chaos," Chaos 17, 043128.

Izhikevich, E. M. [2007] Dynamical Systems in Neuroscience: The Geometry of Excitability and Bursting (MIT Press, Cambridge).

Kurrer, C. \& Schulten, K. [1991] "Effect of noise and perturbations on limit cycle systems," Phys. D 50, $311-320$.

Lindner, B. \& Schimansky-Geier, L. [1999] "Analytical approach to the stochastic FitzHugh-Nagumo system and coherence resonance," Phys. Rev. E 60, 7270-7276.

Longtin, A. [1997] "Autonomous stochastic resonance in bursting neurons," Phys. Rev. E 55, 868-876.

Matsumoto, K. \& Tsuda, I. [1983] "Noise-induced order," J. Stat. Phys. 31, 87-106.

McDonnell, M. D., Stocks, N. G., Pearce, C. E. M. \& Abbott, D. [2008] Stochastic Resonance: From Suprathreshold Stochastic Resonance to Stochastic Signal Quantization (Cambridge University Press).

Milshtein, G. N. \& Ryashko, L. B. [1995] "A first approximation of the quasipotential in problems of the stability of systems with random non-degenerate perturbations," J. Appl. Math. Mech 59, 47-56.

Osipov, V. V. \& Ponizovskaya, E. V. [2000] "Multivalued stochastic resonance in a model of an excitable 
neuron," Phys. Lett. A 271, 191-197.

Pikovsky, A. S. \& Kurths, J. [1997] "Coherence resonance in a noise-driven excitable system," Phys. Rev. Lett. 78, 775-778.

Reinker, S., Puil, E. \& Miura, R. M. [2003] "Resonances and noise in a stochastic Hindmarsh-Rose model of thalamic neurons," Bull Math Biol 65, 641-663.

Ryashko, L., Bashkirtseva, I., Gubkin, A. \& Stikhin, P. [2009] "Confidence tori in the analysis of stochastic 3D-cycles," Mathematics and Computers in Simulation 80, 256269.

Shilnikov, A. \& Kolomiets, M. [2008] "Methods of the qualitative theory for the Hindmarsh-Rose model: A case study a tutorial," Int. J. Bifurcation Chaos 18, 2141-2168.

Storace, M., Linaro, D. \& de Lange, E. [2008] "The Hindmarsh-Rose neuron model: bifurcation analysis and piecewise-linear approximations," Chaos 18, 033128.

Wang, X.-J. [1993] "Genesis of bursting oscillations in the Hindmarsh-Rose model and homoclinicity to a chaotic saddle," Physica D 63, 263-274.

Wang, Y., Wang, Z. D. \& Wang, W. [2000] "Dynamical behaviors of periodically forced Hindmarsh-Rose neural model: The role of excitability and intrinsic stochastic resonance," J. Phys. Soc. Jpn. 69, $276-283$.

Wang, Z., Xu, Y. \& Yang, H. [2016] "Levy noise induced stochastic resonance in an FHN model," Science China Technological Sciences 59, 371-375.

Shi, X. \& Lu, Q.-S. [2005] "Coherence resonance and synchronization of Hindmarsh-Rose neurons with noise," Chinese Physics 14, 1088-1094.

Ji, Y. \& Bi, Q.-S. [2011] "Subhopf/fold-cycle bursting in the Hindmarsh-Rose neuronal model with periodic stimulation," Chin. Phys. Lett 28, 090201. 